\title{
Systèmes Différentiels Fuchsiens le Long d'une Sous-Variété
}

\author{
par \\ Yves LAURent* et Teresa Monteiro Fernandes**
}

\section{French Abstract}

Soit $X$ une variété analytique complexe et $Y$ une sous-variété lisse de $X$. Nous introduisons la notion de "système differentiel fuchsien le long de $Y$ ", et nous montrons que pour de tels systèmes les solutions méromorphes et les solutions à singularités éssentielles sont les mêmes. Nous montrons aussi que les solutions formelles (le long de $Y$ ) sont toujours convergentes. Ces résultats sont bien connus dans le cas holonôme singulier régulier.

\section{English Abstract}

Let $X$ be a complex analytic manifold and let $Y$ be a submanifold of $X$. We introduce the notion of "fuchsian differential system along $Y$ ", and we prove that for such systems the meromorphic solutions and those with essential singularities are the same. We prove as well that the formal solutions (along $Y$ ) always converge. These results are well-known in the regular holonomic case.

\section{$\S 0$. Introduction}

$\S 1$. Construction de la variété 1 -microcaractéristique relative

1. 1. Filtrations sur $\mathscr{D}_{X}$.

1.2. Variété caractéristique relative.

1.3. Variété 1-microcaractéristique relative.

$\S 2$. Théorèmes de comparaison

2. 1. Définition des $\mathscr{D}_{X}$-modules fuchsiens.

2.2. Théorème de comparaison pour $B_{Y \mid X}$ et $B_{Y \mid X}^{\infty}$.

2.3. Théorème de comparaison pour $\mathcal{O}_{X \mid Y}$ et $\mathcal{O}_{X}$.

\section{$\S 3$. Applications}

3. 1. Comparaison des cohomologies d'un système fuchsien.

3. 2. Systèmes induits. Problème de Gauchy.

Communicated by M. Kashiwara, October 12, 1987.

* Université Paris-Sud, Département de Mathématique, Bât 425, 91405 Orsay, France.

** Faculdade de Ciências da Universidade de Lisboa, Dept ${ }^{\circ}$. Matemática, Bloco $\mathrm{C} 1,3^{\circ}$. Andar, Rua Ernesto Vasconcelos, 1700 Lisboa, Portugal et CMAF, INIC, Complexo II, 2, Av. Prof. Gama Pinto, 1699 Lisboa Codex, Portugal. 


\section{§ 0. Introduction}

Soient $X$ une variété analytique complexe et $Y$ une sous-variété lisse de $X$. Soient $\mathscr{D}_{X}$ (resp. $\mathscr{D}_{X}^{\infty}$ ) le faisceau des opérateurs différentiels d'ordre fini (resp. d'ordre infini) sur $X, \mathcal{O}_{X}$ le faisceau des fonctions holomorphes sur $X$ et $\mathcal{O}_{X \hat{Y} Y}$ le completé formel de $\mathcal{O}_{X}$ le long de $Y$. Soit $B_{Y \mid X}$ (resp. $B_{Y \mid X}^{\infty}$ ) le faisceau des hyperfonctions holomorphes le long de $Y$ d'ordre fini (resp. d'ordre infini).

Dans ce travail nous introduisons la notion de "module fuchsien le long de $Y$ " et nous démontrons que pour un tel $\mathscr{D}_{X}$-module on a

$$
\mathscr{D}_{X}^{\infty} \underset{\mathscr{D}_{X}}{\otimes} \boldsymbol{R} \Gamma_{[Y]}(\mathscr{M}) \simeq \boldsymbol{R} \Gamma_{Y}\left(\mathscr{D}_{X}^{\infty} \underset{\mathscr{D}_{X}}{\otimes} \mathscr{M}\right)
$$

ainsi que les théorèmes dits de "comparaison" entre les solutions formelles et les solutions convergentes, i. e.

$$
\left.\boldsymbol{R} \mathscr{H} o m_{\mathscr{D}_{X}}\left(\mathscr{M}, \mathcal{O}_{X}\right)\right|_{Y} \simeq \boldsymbol{R} \mathscr{H} o m_{\mathscr{D}_{X}}\left(\mathscr{M}, \mathcal{O}_{X \hat{Y}}\right)
$$

et entre les solutions méromorphes et les solutions à singularités éssentielles;

$$
\boldsymbol{R} \mathscr{H} o m_{\mathscr{D}_{X}}\left(\mathscr{M}, B_{Y \mid X}\right) \simeq \boldsymbol{R} \mathscr{H} o_{\mathscr{D}_{X}}\left(\mathscr{M}, B_{Y \mid X}^{\infty}\right) .
$$

Ces théorèmes sont bien connus dans le cas où $\mathscr{M}$ est un module holonôme à singularités régulières (of. $[\mathrm{K}-\mathrm{K}-2]$, voir aussi [M], $[R])$.

Pour définir la notion de module fuchsien nous construisons une variété 1-microcaractéristique relative.

Rappelons que la variété 1-microcaractéristique d'un $\mathscr{D}_{X}$-module $\mathscr{M}$ le long du fibré conormal $\Lambda^{*}$ à $Y$ a été définie dans [M. F.] où elle est notée $C_{\Lambda^{*}}^{1}(\mathscr{M})$ et dans [L] où elle est notée $C_{\Lambda^{*}}(\infty, 1)(\mathscr{M})$.

Cette variété 1-microcaractéristique était un sous-ensemble de $T^{*} \Lambda^{*}$ alors qu'ici la variété 1-microcaractéristique relative est un sousensemble du fibré cotangent relatif $T^{*}(\Lambda \mid Y)$ (où $\Lambda$ désigne le fibré normal $\left.T_{Y} X\right)$.

Nous disons qu'un module est fuchsien le long de $Y$ si sa variété 1-microcaractéristique relative ne rencontre pas le fibré conormal à la sphère unité des fibres de $\Lambda$ au dessus de $Y$.

Par exemple si le module $\mathscr{M}$ est de la forme $\mathscr{D}_{X} / \mathscr{D}_{X} P$ et si $Y=\left\{\left(y_{1}, \ldots, y_{p}, t\right) \in C^{p+1} ; t=0\right\}$, il sera fuchsien le long de $Y$ si et 
seulement si $P$ s'écrit sous la forme

$$
P\left(y, t, D_{y}, D_{t}\right)=t^{m} D_{t}^{n}\left[P_{0}\left(y, t D_{t}\right)+t Q\left(y, D_{y}, t, t D_{t}\right)\right]
$$

où $Q$ est d'ordre inférieur ou égal à l'ordre de $P_{0}$ tandis que l'ordre de $P_{0}$ est égal à celui de $P_{0}\left(0, t D_{t}\right)$.

Ainsi l'opérateur $\left(t D_{t}\right)^{2}+\dot{\phi}(y)+t D_{y}$ est fuchsien le long de $Y$ alors que l'opérateur

$$
\left(t D_{t}\right)^{2}+D_{y} \text { ne l'est pas. }
$$

Signalons que dans le cas où $Y$ est une hypersurface et où $\mathscr{M}$ se réduit à une seule équation le théorème de comparaison entre les solutions dans $\mathcal{O}_{X}$ et dans $\mathcal{O}_{X \hat{Y} Y}$ a été démontré par Oshima ([O]).

Lorsque $Y$ est réduit à un point la condition sur $P$ est celle énnoncée par Kashiwara, Kawai et Sjöstrand dans [K-K-S] et par ailleurs les systèmes qui admettent une $b$-fonction régulière relative à $Y$ au sens de [K-3], [S], sont fuchsiens le long de $Y$. (En particulier les modules holonômes réguliers sont fuchsiens le long de toute sousvariété de $X$ ).

Notre méthode de démonstration consiste à démontrer les théorèmes de comparaison d'abord pour un opérateur et ensuite pour un système.

De ces théorèmes, nous déduisons l'isomorphisme

$$
R \Gamma_{Y}\left(\mathscr{D}_{X}^{\infty}{\underset{\mathscr{D}}{X}}_{X} \mathscr{M}\right) \simeq \mathscr{D}_{X}^{\infty} \otimes_{\mathscr{D}_{X}} R \Gamma_{[Y]}(\mathscr{M})
$$

puis que les modules induits par $\mathscr{M}$ sur $Y$ sont les mêmes qu'on les prennent au sens des opérateurs d'ordre fini ou d'ordre infini c'est à dire

$$
\mathscr{D}_{Y}^{\infty} \otimes_{\mathscr{D}_{Y}} \mathscr{M}_{Y} \simeq\left(\mathscr{D}_{X}^{\infty} \otimes_{\mathscr{D}_{X}}^{\otimes} \mathscr{M}\right)_{Y}
$$

Enfin nous obtenons un théorème du type Cauchy-Kowalewsky.

Nous tenons à remercier P. Schapira qui nous a soumis ce problème et $M$. Kashiwara avec qui nous avons tenu de très utiles discussions.

\section{§1. Construction de la Variété 1-Microscaractéristique Relative}

\section{1. Filtrations sur $\mathscr{D}_{X}$}

Commençons par rappeler quelques notions sur les filtrations que 
l'on peut définir sur l'anneau des opérateurs différentiels (voir [K-3], [S], [L. Sch.]).

Soient $X$ une variété analytique complexe, $Y$ une sous-variété lisse de $X, \mathcal{O}_{X}$ le faisceau des fonctions holomorphes sur $X$ et $\mathscr{J}_{Y}$ l'idéal de définition de $Y$ dans $\mathcal{O}_{X}$. On note $\Lambda=T_{Y} X$ le fibré normal à $Y$ et $p: \Lambda \rightarrow Y$ la projection. Soit $\mathscr{D}_{X}$ le faisceau d'anneaux des opérateurs différentiels d'ordre fini sur $X$. Sur $\mathscr{D}_{X}$ on définit deux filtrations :

(i) La filtration usuelle par l'ordre que l'on notera $F_{.}\left(\mathscr{D}_{X}\right)$ ou $\left(\mathscr{D}_{X, j}\right)_{j \in N}$.

(ii) La filtration associée à $Y$ que l'on notera $V \cdot\left(\mathscr{D}_{X}\right)$, par définition on a :

$$
V_{k}\left(\mathscr{D}_{X}\right)=\left\{P \in \mathscr{D}_{X}, P \mathscr{J}_{Y}^{j} \subset \mathscr{J}_{Y}^{j-k} \quad \text { for any } j \in \mathbb{Z}\right\}
$$

avec la convention $\mathscr{J}_{Y}^{j}=\mathcal{O}_{X}$ si $j \leq 0$.

Le gradué $g r_{V}\left(\mathscr{D}_{X}\right)$ est alors isomorphe à $p_{*} \mathscr{D}_{[\Lambda]}$ où $\mathscr{D}_{[A]}$ désigne le sous-faisceau d'anneaux de $\mathscr{D}_{\Lambda}$ des opérateurs différentiels sur $\Lambda$ à coefficients polynomiaux dans les fibres de $p$.

On déduit une bi-filtration sur $\mathscr{D}_{X}$

$$
V_{k} F_{l}\left(\mathscr{D}_{X}\right)=V_{k}\left(\mathscr{D}_{X}\right) \cap F_{l}\left(\mathscr{D}_{X}\right) \text {. }
$$

Suivant [S] on note $R_{V F}\left(\mathscr{D}_{X}\right)$ l'anneau de Rees associé

$$
R_{V F}\left(\mathscr{D}_{X}\right)=\underset{\substack{k \in \in \mathbb{Z} \\ l \in N}}{\bigoplus} V_{k} F_{l}\left(\mathscr{D}_{X}\right) \theta^{k} \tau^{l} .
$$

C'est un faisceau d'anneaux cohérent et noethérien. Soit $\mathscr{M}$ un $\mathscr{D}_{X}$-module cohérent. Une bi-filtration de $\mathscr{M}$ est une famille $\left(\mathscr{M}_{i j}\right)_{\substack{i \in Z \\ j \in N}}$ de sous- $\mathcal{O}_{X}$-modules cohérents de $\mathscr{M}$ vérifiant:

$$
\cup_{i, j} \mathscr{M}_{i j}=\mathscr{M} \text {. }
$$

Pour tous $i, k \in \boldsymbol{Z}, j, l \in \boldsymbol{N}$ on a

$$
V_{k} F_{l}\left(\mathscr{D}_{X}\right) \mathscr{M}_{i j} \subset \mathscr{M}_{i+k, j+l} \text {. }
$$

Définition 1.1.1. Une bi-filtration sur $\mathscr{M}$ est bonne si localement il existe des sections $u_{1}, \ldots u_{N}$ de $\mathscr{M}$ et $\left(k_{1}, \ldots, k_{N}\right) \in Z^{N},\left(l_{1}, \ldots, l_{N}\right) \in N^{N}$ tels que l'on ait

$$
\mathscr{M}_{k l}=\sum_{i=1}^{N} V_{k-k_{i}} F_{l-l_{i}}\left(\mathscr{D}_{X}\right) u_{i} .
$$

A toute bi-filtration $\mathscr{M}_{i j}$ on associe le module de Rees $R(\mathscr{M})=$ 
$\bigoplus_{i \in Z} \mathscr{M}_{i j} \theta^{i} \tau^{j}$ qui est un $R_{V F}\left(\mathscr{D}_{X}\right)$-module, cohérent si et seulement si la $\underset{\substack{i \in Z \\ j \in N}}{ }$ bi-filtration est bonne.

On a le lemme d'Artin-Rees:

Lemme 1.1.2. Soit $\left(\mathscr{M}_{i j}\right)_{\substack{i \in Z \\ j \in N}}$ une bonne br-filtration d'un $\mathscr{D}_{X}$-module cohérent $\mathscr{M}$. Alors elle induit sur tout sous-quotient de $\mathscr{M}$ une bonne bifiltration.

\subsection{Variété caractéristique relative}

La variété caractéristique relative d'un $\mathscr{D}_{x}$-module est définie par Schapira dans [Sch]. Ici nous allons la définir pour un module sur l'anneau de Rees de $\mathscr{D}_{X}$. Lorsque ce module est le module de Rees d'un $\mathscr{D}_{X}$-module on retrouve la définition de [Sch].

Soit $Y$ une variété analytique complexe et soit $\Lambda \stackrel{p}{\longrightarrow} Y$ un fibré vectoriel. On définit le fibré cotangent relatif $T^{*}(\Lambda \mid Y)$ par la suite exacte de fibrés sur $Y$ :

$$
0 \rightarrow \underset{Y}{\Lambda} T^{*} Y \rightarrow T^{*} \Lambda \rightarrow T^{*}(\Lambda \mid Y) \rightarrow 0
$$

et les projections canoniques

$$
\tilde{p}: T^{*} \Lambda \rightarrow Y, \quad \widetilde{p}: T^{*}(\Lambda \mid Y) \rightarrow Y, \gamma: T^{*} \Lambda \rightarrow \Lambda .
$$

On note $\mathcal{O}_{[\Lambda]}$ le sous-faisceau de $\mathcal{O}_{\Lambda}$ des sections polynomiales dans les fibres de $p$. Soit $\mathscr{D}_{[A]}$ l'anneau des opérateurs différentiels sur $\Lambda$ polynomiaux dans les fibres de $p$.

On note encore $\mathscr{D}_{[\Lambda \mid Y]}$ le sous-anneau de $\mathscr{D}_{[\Lambda]}$ des opérateurs relatifs, c'est à dire, des opérateurs qui commutent avec $p^{-1} \mathcal{O}_{Y}$.

On note $\mathscr{D}_{[\Lambda], j}$ (resp. $\mathscr{D}_{\left[\Lambda_{1} Y\right], j}$ ) la filtration de $\mathscr{D}_{[\Lambda]}$ (resp. de $\left.\mathscr{D}_{[\Lambda \mid Y]}\right)$ par l'ordre. Le gradué associé s'identifie à $\gamma_{*} \mathcal{O}_{\left[T^{*} A\right]}$, le faisceau des fonctions holomorphes sur $T^{*} \Lambda$ polynomiales dans les fibres de $\tilde{p} T^{*} \Lambda \stackrel{\tilde{p}}{\longrightarrow} Y$ (resp. à $\gamma_{*} \mathcal{O}_{\left[T^{*}(\Lambda \mid Y)\right]}$, le faisceau des fonctions holomorphes sur $T^{*}(\Lambda \mid Y)$ polynomiales dans les fibres de $\left.\tilde{p}\right)$.

Notons $\mathscr{A}=\bigoplus_{j \in N} \mathscr{D}_{[\Lambda],} \tau^{j}$ l'anneau de Rees de $\mathscr{D}_{[A]}$ et $\mathscr{A}_{0}=\bigoplus_{, \in N}$ $\mathscr{D}_{[\Lambda ; Y], j} \tau^{j}$ l'anneau de Rees de $\mathscr{D}_{[\Lambda ! Y]}$.

On a alors $\mathscr{A} / \tau \mathscr{A} \simeq \operatorname{gr}\left(\mathscr{D}_{[\Lambda]}\right) \simeq \gamma_{*} \mathcal{O}_{\left.\left[T^{*}\right]\right]}$ et de même $\mathscr{A}_{0} / \tau \mathscr{A}_{0} \simeq$ $\operatorname{gr}\left(\mathscr{D}_{[\Lambda \mid Y]}\right) \simeq \gamma_{*} \mathcal{O}_{\left[T^{*}(\Lambda \mid Y)\right]}$. 
Proposition 1.2.1. Tout $\mathscr{A}$-module cohérent est un $\mathscr{A}_{0}$-module pseudocohérent.

\section{Démonstration.}

$\mathscr{A}$ et $\mathscr{A}_{0}$ sont des faisceaux d'anneaux noethériens. Considerons des coordonnées locales $(y, t)$ sur $\Lambda$ où $y=\left(y_{1} \ldots y_{p}\right)$ est un système de coordonnées locales sur $Y$ et $t=\left(t_{1} \ldots t_{q}\right)$ sont linéaires dans les fibres.

Filtrons $\mathscr{D}_{[\Lambda]}$ par $G_{k}\left(\mathscr{D}_{[\Lambda]}\right)=\left\{P \in \mathscr{D}_{[\Lambda]}\right.$, ordre de $P$ en $D_{y}$ est inférieur ou égal à $k\}$ et filtrons $\mathscr{A}$ par $\mathscr{A}_{k}=\operatorname{Rees}\left(G_{k}\left(\mathscr{D}_{[\Lambda]}\right)\right)$, où l'on considère $G_{k}\left(\mathscr{D}_{[A]}\right)$ filtré par l'ordre usuel. Alors $\mathscr{A}_{k} \simeq \mathscr{A}_{0} \otimes_{C} C$ $\left[\tau D_{y}\right]_{k}$ (où $C\left[\tau D_{y}\right]_{k}$ designe l'anneau des polynomes en $\tau D_{y}$ de degré $\leq k)$ et la filtration $\left(\mathscr{A}_{k}\right)_{k \geq 0}$ satisfait les conditions de la proposition 1. 1.5. de $[\mathrm{K}-\mathrm{K}-2]$.

q. e. d.

Soit $\mathscr{N}$ un $\mathscr{A}_{0}$-module cohérent.

Définition 1.2.2. La variété caractéristique de $\mathscr{N}$ (notée $\operatorname{Car}(\mathcal{N})$ ) est

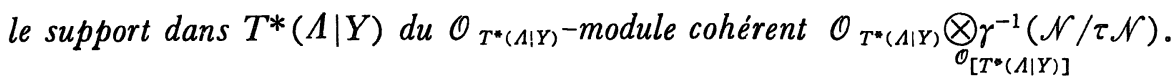

$\operatorname{Car}(\mathscr{N})$ est donc un sous-ensemble analytique fermé de $T^{*}(\Lambda \mid Y)$.

Remarque. Soit $\mathscr{L}$ un $\mathscr{D}_{\left[{ }_{[\mid Y]}\right]}$-module cohérent muni d'une bonne filtration $\mathscr{L}_{k}$ et soit $\mathscr{N}=\bigoplus_{k \in N} \mathscr{L}_{k} \tau^{k}$ le module de Rees associé.

Alors $\mathscr{N}$ est un $\mathscr{A}_{0}$-module cohérent et la variété $\operatorname{Car}(\mathscr{N})$ définie ci-dessus est égale à la variété caractéristique de $\mathscr{L}$ (car on a $\mathscr{N} / \tau \mathscr{N} \simeq \operatorname{gr}(\mathscr{L}))$.

Lemme 1.2.3. Soit $0 \rightarrow \mathscr{N}^{\prime} \rightarrow \mathscr{N} \rightarrow \mathscr{N}^{\prime \prime} \rightarrow 0$ une suite exacte de $\mathscr{A}_{0^{-}}$ modules cohérents. Alors on a

$$
\operatorname{Car}(\mathscr{N})=\operatorname{Car}\left(\mathscr{N}^{\prime}\right) \cup \operatorname{Car}\left(\mathscr{N}^{\prime \prime}\right)
$$

Démonstration.

On a une suite exacte

$$
\mathscr{T o r}_{1}{ }^{\mathscr{A}_{0}}\left(\mathscr{A}_{0} / \tau \mathscr{A}_{0}, \mathscr{N}^{\prime \prime}\right) \rightarrow \frac{\mathscr{N}^{\prime}}{\tau \mathscr{N}^{\prime}} \rightarrow \frac{\mathscr{N}}{\tau \mathscr{N}} \rightarrow \frac{\mathscr{N}^{\prime \prime}}{\tau \mathscr{N}^{\prime \prime}} \rightarrow 0
$$


Par définition $\mathscr{T} \operatorname{rod}_{1}^{\mathscr{A}_{0}}\left(\mathscr{A}_{0} / \tau \mathscr{A}_{0}, \mathscr{N}^{\prime \prime}\right)$ est le noyau (que nous noterons $\mathscr{K})$ de l'application $\tau: \mathscr{N}^{\prime \prime} \rightarrow \mathscr{N}^{\prime \prime}$. Désignons par $T$ le foncteur exact

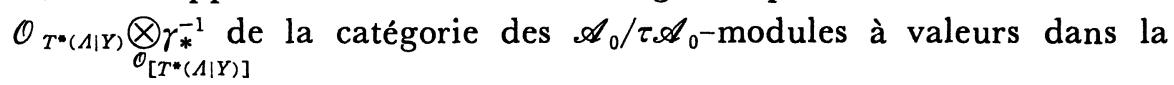
catégorie des $\mathcal{O}_{T^{*}(\Lambda \mid Y)}$-modules $(\operatorname{donc} \operatorname{Car}(\mathscr{N})=\operatorname{supp} T(\mathcal{N} / \tau \mathscr{N})$ ). Pour montrer le lemme il suffit de montrer que si en un point $x^{*} \in T^{*}(\Lambda \mid Y)$ on a $T\left(\mathcal{N}^{\prime \prime} / \tau \mathscr{N}^{\prime \prime}\right)_{x^{*}}=0$ alors $T(\mathscr{K})_{x^{*}}=0$.

Notons $\mathscr{K}^{n}$ le noyau de $\tau^{n}: \mathscr{N}^{\prime \prime} \rightarrow \mathscr{N}^{\prime \prime}$ et $\mathscr{H}^{n}=\mathscr{K}^{n} / \tau \mathscr{K}^{n}$. On a donc $\mathscr{H}^{1}=\mathscr{K}^{1}=\mathscr{K}$. Alors la suite $\mathscr{K}^{1} \subset \mathscr{K}^{2} \subset \ldots \subset \mathscr{K}^{n} \subset \ldots$ est stationnaire dans $\mathscr{N}^{\prime \prime}$ (car $\mathscr{A}_{0}$ est noethérien) et donc il existe $n \in N$ tel que $\mathscr{K}^{n}=\mathscr{K}^{n+1}$, c'est à dire, $\mathscr{H}^{n}=\mathscr{H}^{n+1}$. Or pour tout $p \in N$ on a une suite exacte $\mathscr{H}^{p+1} \rightarrow \mathscr{H}^{p} \rightarrow \mathscr{N}^{\prime \prime} / \tau \mathscr{N}^{\prime \prime}$.

Par suite si $T\left(\mathscr{N}^{\prime \prime} / \tau \mathscr{N}^{\prime \prime}\right)_{x^{*}}=0, \tau$ définit pour tout $p$ un morphisme surjectif de $T\left(\mathscr{H}^{p+1}\right)$ dans $T\left(\mathscr{H}^{p}\right)$ et donc $\tau^{n}$ est un morphisme surjectif de $T\left(\mathscr{H}^{n+1}\right)_{x^{*}}$ dans $T\left(\mathscr{H}^{1}\right)_{x^{*}}=T(\mathscr{K})_{x^{*}}$ mais $\tau^{n}$ étant nul sur $T\left(\mathscr{H}^{n}\right)$ il en résulte $T(\mathscr{K})_{x^{*}}=0$.

Soit maintenant $\mathscr{M}$ un $\mathscr{A}$-module cohérent; il existe toujours localement un $\mathscr{A}_{0}$-sous-module cohérent $\mathscr{M}_{0}$ qui l'engendre (cf. Proposition 1.2.1).

\section{Proposition et Définition 1.2.4.}

a) Soit $\mathscr{M}$ un $\mathscr{A}$-module cohérent. L'ensemble analytique $\operatorname{Car}\left(\mathscr{M}_{0}\right)$ ne dépend pas $d u$ choix du $\mathscr{A}_{0}$-sous-module $\mathscr{M}_{0}$ qui l'engendre; on le note $C_{A \mid Y}(\mathscr{M})$ et on l'appelle variété caractéristique relative de $\mathscr{M}$.

b) Soit $0 \rightarrow \mathscr{M}^{\prime} \rightarrow \mathscr{M} \rightarrow \mathscr{M}^{\prime \prime} \rightarrow 0$ une suite exacte de $\mathscr{A}$-modules cohérents. Alors on $a$

$$
C_{\Lambda \mid Y}(\mathscr{M})=C_{\Lambda \mid Y}\left(\mathscr{M}^{\prime}\right) \cup C_{\Lambda \mid Y}\left(\mathscr{M}^{\prime \prime}\right)
$$

Démonstration.

a) Soient $\mathscr{M}_{0}$ et $\mathscr{M}_{0}^{\prime}$ deux $\mathscr{A}_{0}$-sous-modules cohérents de $\mathscr{M}$ qui l'engendrent. Considérons la filtration $\mathscr{A}_{k}$ de $\mathscr{A}$ définie plus haut. Alors il exist $l \in N$ tel que $\mathscr{M}_{0} \subset \mathscr{A}_{1} \mathscr{M}_{0}^{\prime}$ et $\mathscr{A}_{l}$ étant libre en tant que $\mathscr{A}_{0}$-bimodule on déduit grâce au lemme précédent Car $\left(\mathscr{M}_{0}\right) \subset \operatorname{Car}\left(\mathscr{M}_{0}^{\prime}\right.$.)

b) Résulte de la proposition 1.2.1 et du lemme 1.2.3.

Remarque. Si $\tau$ est injectif sur $\mathscr{M}$ alors il existe un $\mathscr{D}_{[\Lambda]}$-module cohérent $\tilde{\mathscr{M}}$ dont $\mathscr{M}$ est le module de Rees et alors $C_{\Lambda \mid Y}(\mathscr{M})$ est la 
variété caractéristique relative de $\tilde{\mathscr{M}}$ (cf. [Sch], Ch. III).

\subsection{Variété 1-microcaractéristique relative}

Considerons la bi-filtration de $\mathscr{D}_{X}$ définie dans $\S 1$ 1. Par définition on a

$$
R_{V F}\left(\mathscr{D}_{X}\right)=\bigoplus_{\substack{k \in Z \\ l \in N}} V_{k} F_{l}\left(\mathscr{D}_{X}\right) \theta^{k} \tau^{l}
$$

et donc

$$
\frac{R_{V F}\left(\mathscr{D}_{X}\right)}{\theta R_{V F}\left(\mathscr{D}_{X}\right)} \simeq \underset{\substack{k \in Z \\ l \in N}}{\bigoplus} \frac{V_{k} F_{l}\left(\mathscr{D}_{X}\right)}{V_{k-1} F_{l}\left(\mathscr{D}_{X}\right)} \theta^{k} \tau^{l}
$$

Or, comme on a vu au paragraphe 1.1.

$\frac{V_{k} F_{l}\left(\mathscr{D}_{X}\right)}{V_{k-1} F_{l}\left(\mathscr{D}_{X}\right)} \simeq \mathscr{D}_{[1], l}[k]$ (ensemble des opérateurs dans $\mathscr{D}_{[1], l}$ homogènes de degré $k$ dans les fibres de $p: \Lambda \rightarrow Y$ ). Donc

$$
\frac{R_{V F}\left(\mathscr{D}_{X}\right)}{\theta R_{V F}\left(\mathscr{D}_{X}\right)} \simeq \bigoplus_{l \in N}\left(\bigoplus_{k \in \mathbb{Z}} \mathscr{D}_{[A], l}[k]\right) \tau^{l} \simeq \bigoplus_{l \in N} \mathscr{D}_{[A], l^{2}} \tau^{l}=\mathscr{A} .
$$

Soit $\mathscr{M}$ un $\mathscr{D}_{X}$-module cohérent muni d'une bonne bi-filtration; le module de Rees $R(\mathscr{M})$ étant cohérent $\frac{R(\mathscr{M})}{\theta R(\mathscr{M})}$ est un $\mathscr{A}$-module cohérent.

\section{Proposition 1.3.2.}

1) La variété $C_{\Lambda \mid Y}\left(\frac{R(\mathscr{M})}{\theta R(\mathscr{M})}\right)$ est un sous-ensemble analytique de $T^{*}(\Lambda \mid Y)$ indépendant du choix de la bonne bi-filtration de $\mathscr{M}$. On le notera

$$
C_{\Lambda \mid Y}^{1}(\mathscr{M}) \text {. }
$$

2) Si $0 \rightarrow \mathscr{M}^{\prime} \rightarrow \mathscr{M} \rightarrow \mathscr{M}^{\prime \prime} \rightarrow 0$ est une suite exacte de $\mathscr{D}_{X}$-modules cohérents alors on a

$$
C_{A \mid Y}^{1}(\mathscr{M})=C_{\Lambda \mid Y}^{1}\left(\mathscr{M}^{\prime}\right) \cup C_{\Lambda \mid Y}^{1}\left(\mathscr{M}^{\prime \prime}\right)
$$

Démonstration.

1) Soient $\mathscr{M}_{i}$, et $\mathscr{M}_{i}^{\prime}$ deux bonnes bi-filtrations sur $\mathscr{M}$. La démonstration se fera en deux étapes:

a) Supposons d'abord que pour tout $i, j, \mathscr{M}_{i}^{\prime} \subset \mathscr{M}_{i j}$ et montrons que dans ce cas 


$$
C_{A \mid Y}\left(\frac{R(\mathscr{M})}{\theta R(\mathscr{M})}\right) \subset C_{\Lambda \mid Y}\left(\frac{R\left(\mathscr{M}^{\prime}\right)}{\theta R\left(\mathscr{M}^{\prime}\right)}\right)
$$

i) Supposons de plus qu'il existe $k \geq 1$ tel que $\mathscr{M}_{i j}^{\prime} \subset \mathscr{M}_{i}, \subset \mathscr{M}_{i+k,}^{\prime}$ pour tous $i, j$. Soit $\mathscr{N}_{i j}=\mathscr{M}_{i j} \cap \mathscr{M}_{i+1, j}^{\prime}$. On a

$$
\begin{aligned}
& \mathscr{M}_{i j}^{\prime} \subset \mathscr{N}_{i j} \subset \mathscr{M}_{i+1, j}^{\prime} \\
& \mathscr{M}_{i-k+1, j} \subset \mathscr{N}_{i}, \subset \mathscr{M}_{i j} .
\end{aligned}
$$

Donc en raisonnant par récurrence sur $k$ on peut se ramener au cas $k=1$. On a alors

$\theta R(\mathscr{M}) \subset R\left(\mathscr{M}^{\prime}\right) \subset R(\mathscr{M})$ et donc les suites exactes de $\mathscr{A}$-modules cohérents

$$
\begin{aligned}
0 & \rightarrow \frac{\theta R(\mathscr{M})}{\theta R\left(\mathscr{M}^{\prime}\right)} \rightarrow \frac{R\left(\mathscr{M}^{\prime}\right)}{\theta R\left(\mathscr{M}^{\prime}\right)} \rightarrow \frac{R\left(\mathscr{M}^{\prime}\right)}{\theta R(\mathscr{M})} \rightarrow 0 \\
0 & \rightarrow \frac{R\left(\mathscr{M}^{\prime}\right)}{\theta R(\mathscr{M})} \rightarrow \frac{R(\mathscr{M})}{\theta R(\mathscr{M})} \rightarrow \frac{R(\mathscr{M})}{R\left(\mathscr{M}^{\prime}\right)} \rightarrow 0 .
\end{aligned}
$$

En appliquant la proposition 1.2.4. on obtient

$$
C_{\Lambda, Y}\left(\frac{R\left(\mathscr{M}^{\prime}\right)}{\theta R\left(\mathscr{M}^{\prime}\right)}\right)=C_{\Lambda \mid Y}\left(\frac{R(\mathscr{M})}{\theta R(\mathscr{M})}\right) .
$$

ii) On suppose seulement $\mathscr{M}_{i,}^{\prime} \subset \mathscr{M}_{i}$. Notons $\mathscr{M}_{k s}^{\prime \prime}=\mathscr{M}_{k} \cap\left(\underset{i \in Z}{\cup} \mathscr{M}_{i j}^{\prime}\right)$. On a donc :

$$
R\left(\mathscr{M}^{\prime \prime}\right)=R(\mathscr{M}) \cap\left(\cup_{i \geq 0} \theta^{-i} R\left(\mathscr{M}^{\prime}\right)\right) .
$$

Soit $\mathscr{L}_{k}=R(\mathscr{M}) \subset\left(\bigcup_{0 \leq i \leq k} \theta^{-i} R\left(\mathscr{M}^{\prime}\right)\right)$; c'est une suite croissante de $R_{V F}\left(\mathscr{D}_{X}\right)$ sous-modules cohérents de $R(\mathscr{M})$, donc elle est localement stationnaire et il existe (localement) un entier $r \geq 0$ tel que

$$
R\left(\mathscr{M}^{\prime \prime}\right)=R(\mathscr{M}) \cap \theta^{-r} R\left(\mathscr{M}^{\prime}\right) .
$$

En particulier $R\left(\mathscr{M}^{\prime \prime}\right)$ est cohérent donc $\mathscr{M}_{i j}^{\prime \prime}$ est une bonne bi-filtration de $\mathscr{M}$.

On a $\theta^{r} R\left(\mathscr{M}^{\prime \prime}\right) \subset R\left(\mathscr{M}^{\prime}\right) \subset R\left(\mathscr{M}^{\prime \prime}\right)$.

et d'après le cas i)

$$
C_{\Lambda \mid Y}\left(\frac{R\left(\mathscr{M}^{\prime}\right)}{\theta R\left(\mathscr{M}^{\prime}\right)}\right)=C_{\Lambda \mid Y}\left(\frac{R\left(\mathscr{M}^{\prime \prime}\right)}{\theta R\left(\mathscr{M}^{\prime \prime}\right)}\right)
$$

Par ailleurs l'application canonique

$$
\frac{R\left(\mathscr{M}^{\prime \prime}\right)}{\theta R\left(\mathscr{M}^{\prime \prime}\right)} \rightarrow \frac{R(\mathscr{M})}{\theta R(\mathscr{M})} \text { est injective }
$$


donc

$$
C_{\Lambda \mid Y}\left(\frac{R\left(\mathscr{M}^{\prime}\right)}{\theta R\left(\mathscr{M}^{\prime}\right)}=C_{\Lambda \mid Y}\left(\frac{R\left(\mathscr{M}^{\prime \prime}\right)}{\theta R\left(\mathscr{M}^{\prime \prime}\right)}\right) \subset C_{\Lambda \mid Y}\left(\frac{R(\mathscr{M})}{\theta R(\mathscr{M})}\right) .\right.
$$

b) Cas général: Soient $\mathscr{M}_{i j}$ et $\mathscr{M}_{i j}^{\prime}$ deux bonnes bi-filtrations sur $\mathscr{M}$. Alors il existe $k_{0}, l_{0} \in N$ tels que

$$
\mathscr{M}_{i j} \subset \mathscr{M}_{i+k_{0}, j+l_{0}}^{\prime} \quad \forall i, j
$$

et en notant $\mathscr{M}_{i j}^{\prime \prime}=\mathscr{M}_{i+k_{0}, j+l_{0}}^{\prime}$ on se retrouve dans la situation de a) c'est à dire, $C_{\Lambda \mid Y}^{1}(\mathscr{M}) \subset C_{\Lambda \mid Y}^{1}\left(\mathscr{M}^{\prime \prime}\right)$ et il est clair que $C_{\Lambda \mid Y}^{1}\left(\mathscr{M}^{\prime \prime}\right)=C_{\Lambda \mid Y}^{1}$ $\left(\mathscr{M}^{\prime}\right)$. De la même manière on conclut que $C_{\Lambda \mid Y}^{1}\left(\mathscr{M}^{\prime}\right)$ est contenu dans $C_{\Lambda \mid Y}^{1}(\mathscr{M})$.

q. e. d.

2) Soit $0 \rightarrow \mathscr{M}^{\prime} \rightarrow \mathscr{M} \rightarrow \mathscr{M}^{\prime \prime} \rightarrow 0$ une suite exacte de $\mathscr{D}_{X}$-modules cohérents. Soit $\mathscr{M}_{i j}$ une bonne bi-filtration de $\mathscr{M}$ et considerons $\mathscr{M}^{\prime}$ (resp. $\mathscr{M}^{\prime \prime}$ ) muni de la bi-filtration induite (resp. de la bi-filtration quotient).

On a alors les suites exactes

$$
\begin{aligned}
& 0 \rightarrow R\left(\mathscr{M}^{\prime}\right) \rightarrow R(\mathscr{M}) \rightarrow R\left(\mathscr{M}^{\prime \prime}\right) \rightarrow 0 \\
& 0 \rightarrow \frac{R\left(\mathscr{M}^{\prime}\right)}{\theta R\left(\mathscr{M}^{\prime}\right)} \rightarrow \frac{R(\mathscr{M})}{\theta R(\mathscr{M})} \rightarrow \frac{R\left(\mathscr{M}^{\prime \prime}\right)}{\theta R\left(\mathscr{M}^{\prime \prime}\right)} \rightarrow 0
\end{aligned}
$$

et il reste à appliquer la proposition 1.2.4.

q. e. d.

Soit $P$ un opérateur différentiel et soit $\hat{\sigma}_{\Lambda}(P)$ le symbole principal de $P$ au sens de la $V$-filtration.

Définition 1.3.3. On définit

$$
\sigma_{\Lambda \mid Y}^{1}(P)=\sigma\left(\hat{\sigma}_{\Lambda}(P)\right) \text { si } \hat{\sigma}_{\Lambda}(P) \in \mathscr{D}_{[\Lambda \mid Y]} \text { et si l'ordre }
$$
de $\hat{\sigma}_{\Lambda}(P)$ est égal à l'ordre de $P$ (pour la F-filtration), et

$$
\sigma_{\Lambda \mid Y}^{1}(P)=0 \text { dans les autres cas. }
$$

Dans un système de coordonnées locales $(y, t)$ sur $X$ où $Y$ est donnée par $\{t=0\}$ dire que $\sigma_{\Lambda \mid Y}^{1}(P)$ est non nul signifie que $P$ s'écrit sous la forme

$$
P\left(y, t, D_{y}, D_{t}\right)=P_{0}\left(y, t, D_{t}\right)+P_{1}\left(y, t, D_{y}, D_{t}\right)
$$

avec ordre $P_{0}=$ ordre $P$ au sens de la $F$-filtration et ordre $P_{1}$ strictement plus petit que celui de $P$ au sens de la $V$-filtration. Alors 
$\sigma_{\Lambda \mid Y}^{1}(P)=\sigma\left(P_{0}\right)$

Proposition 1.3.4. Soit $\mathscr{I}$ un idéal cohérent de $\mathscr{D}_{X}$; alors:

$$
C_{\Lambda \mid Y}^{1}\left(\mathscr{D}_{X} / \mathscr{I}\right)=\left\{\omega \in T^{*}(\Lambda \mid Y), \forall P \in \mathscr{I}, \sigma_{\Lambda \mid Y}^{1}(P)(\omega)=0\right\} .
$$

Démonstration.

Considerons $\mathscr{M}=\mathscr{D}_{X} / \mathscr{I}$ muni de la bonne bi-filtration $\mathscr{M}_{i}=\mathscr{D}_{i j} / \mathscr{D}_{i j}$ $\cap \mathscr{I}$ où $\mathscr{D}_{i j}=V_{i} F_{j}\left(\mathscr{D}_{X}\right)$. Alors

$$
\frac{R(\mathscr{M})}{\theta R(\mathscr{M})}=\bigoplus_{i, j} \frac{\mathscr{D}_{i j}}{\left(\mathscr{D}_{i j} \cap \mathscr{I}\right)+\mathscr{D}_{i-1, j}} \theta^{i} \tau^{j}
$$

Or on a

$$
\frac{\mathscr{D}_{i j}}{\left(\mathscr{D}_{i j} \cap \mathscr{I}\right)+\mathscr{D}_{i-1, j}} \simeq \mathscr{D}_{[\Lambda], j}[i] / \tilde{\mathscr{I}}_{i j} \text { où } \tilde{\mathscr{I}}_{i j}=\hat{\sigma}_{\Lambda}\left(\mathscr{I} \cap \mathscr{D}_{i j}\right) .
$$

Soit $\overline{\mathscr{I}}_{i}=\bigoplus_{j \geq 0} \tilde{\mathscr{I}}_{i j} ;$ alors $\tilde{\mathscr{I}}=\bigcup_{j \geq 0} \tilde{\mathscr{I}}_{j}$ est un idéal gradué de $\mathscr{D}_{[\Lambda]}$, la famille $\tilde{\mathscr{I}}_{j}$ definit une filtration de $\tilde{\mathscr{I}}$ et on note $R(\tilde{\mathscr{I}})=\bigoplus_{j \geq 0} \tilde{\mathscr{I}}_{j} \tau^{j}$ l'anneau de Rees associé. (Remarquons que cette filtration n'est pas en géneral la filtration induite par celle de $\left.\mathscr{D}_{[A]}\right)$.

Alors

$$
\frac{R(\mathscr{M})}{\theta R(\mathscr{M})} \simeq \frac{R\left(\mathscr{D}_{[A]}\right)}{R(\tilde{\mathscr{I}})}
$$

et le module $\mathscr{M}_{0}=R\left(\mathscr{D}_{\left[\Lambda_{i} Y\right]}\right) / R(\tilde{\mathscr{I}}) \cap R\left(\mathscr{D}_{[\Lambda \mid Y]}\right)=\frac{R\left(\mathscr{D}_{[\Lambda \mid Y]}\right)}{R\left(\tilde{\mathscr{I}} \cap \mathscr{D}_{[\Lambda \mid Y]}\right)}$ est cohérent sur $R\left(\mathscr{D}_{[\Lambda \mid Y]}\right)$ et engendre $R(\mathscr{M}) / \theta R(\mathscr{M})$.

Notons $\sigma_{j}(Q)$ le symbole d'ordre $j$ de $Q$ pour la $F$-filtration, c'est à dire l'image de $Q$ dans $F_{j}\left(\mathscr{D}_{[\Lambda \mid Y]}\right) / F_{j-1}\left(\mathscr{D}_{[\Lambda \mid Y]}\right)$. (En particulier si l'ordre de $Q$ est inférieur à $j, \sigma,(Q)=0)$.

Alors $\operatorname{Car}\left(\mathscr{M}_{0}\right)=\bigcup\left\{\omega \in T^{*}(\Lambda \mid Y) \mid \sigma_{j}(Q)(\omega)=0\right.$ pour tout $Q \in \tilde{\mathscr{I}} \cap$ $\left.\mathscr{D}_{[\Lambda \mid Y]}\right\}$.

Finalement

$\operatorname{Car}\left(\mathscr{M}_{0}\right)=\left\{\omega \in T^{*}(\Lambda \mid Y), \sigma(Q)(\omega)=0\right.$ pour tout $Q \in \mathscr{D}_{[\Lambda \mid Y]}$ tel qu'il existe $P \in \mathscr{I}, Q=\hat{\sigma}_{\Lambda}(P)$ et ordre de $Q=\operatorname{ordre}$ de $\left.P\right\}$ q. e. d.

Remarque: Dans le cas absolu, c'est à dire, si on calcule la variété caractéristique de $\frac{R(\mathscr{M})}{\theta R(\mathscr{M})}$ dans $T^{*} \Lambda$ on retrouve la variété $C_{\Lambda^{*}}^{1}(\mathscr{M})$ 
définie par les auteurs (cf. [L], [M. F.]), où $\Lambda^{*}$ désigne le fibré conormal $T_{Y}^{*} X$ à $Y$ dans $X$.

Exemple.

Considérons l'opérateur $t^{2} D_{t}+1$ pour $X=C$ et $Y=\{0\}$.

Alors suivant les notations de la démonstration précédente on a

$$
\begin{aligned}
& \tilde{\mathscr{I}}_{0}=0, \tilde{\mathscr{I}}_{j}=\mathscr{D}_{[\Lambda], j-1}, j \geq 1 \text { donc } \\
& \frac{R\left(\mathscr{D}_{[\Lambda]}\right)}{R(\tilde{\mathscr{I}})} \simeq \bigoplus_{j} \mathcal{O}_{\left[T^{*} \Lambda\right]}(j) \tau^{j} \text { et donc } \\
& C_{\Lambda \mid Y}^{1}\left(\frac{\mathscr{D}_{c}}{\mathscr{D}_{c}\left(t^{2} D_{t}+1\right)}\right)=T^{*} \Lambda .
\end{aligned}
$$

\section{§2. Théorèmes de Comparaison}

\subsection{Définition des $\mathscr{D}_{X}$-modules fuchsiens}

Soit $X$ une variété analytique complexe et $Y$ une sous-variété lisse. Comme au $\S 1$ on notera $\Lambda=T_{Y} X$ et $p: T_{Y} X \rightarrow Y$ la projection. Soit $y_{0}$ un point de $Y$ et $\sum=p^{-1}\left(y_{0}\right) \subset \Lambda$.

Si $S$ est une sous-variété analytique réelle de $\sum$ le fibré conormal $T_{S}^{*} \sum$ à $S$ dans $\sum$ est un sous-ensemble du fibré cotangent réel $\left(T^{*} \sum\right)_{R}$ que l'on identifiera au fibré cotangent complexe $T^{*} \sum$.

Ce dernier fibré est isomorphe à $T^{*}(\Lambda \mid Y) \times \underset{\Lambda}{\sum}$ et peut donc être considéré comme un sous-ensemble de $T^{*}(\Lambda \mid Y)$. On peut donc définir

Définition 2.1.1. Soit $\mathscr{M}$ un $\mathscr{D}_{X}$-module cohérent défini au voisinage d'un point $y_{0} \in Y$. On dira que $\mathscr{M}$ est fuchsien le long de $Y$ en $y_{0}$ s'il existe une métrique hermitienne sur l'espace vectoriel $\sum=p^{-1}\left(y_{0}\right)$ dont la sphère unite $S$ vérifie

$$
T_{S}^{*} \sum \cap C_{\Lambda \mid Y}^{1}(\mathscr{M}) \subset\{0\} .
$$

On dira que $\mathscr{M}$ est fuchsien le long de $Y$ sur un ouvert $U$ de $Y$ s'il est fuchsien le long de $Y$ en tout point de $U$.

\section{Remarques.}

$1-\mathrm{Si} \mathscr{M}$ est fuchsien le long de $Y$ en $y_{0}$ il existe un voisinage $U$ de 
$y_{0}$ dans $Y$ tel que $\mathscr{M}$ soit fuchsien le long de $Y$ sur $U$.

2-La définition 2.1.1 dépend du choix de la métrique, en particulier si on a une suite exacte de $\mathscr{D}_{X}$-modules cohérents

$$
0 \rightarrow \mathscr{M}^{\prime} \rightarrow \mathscr{M} \rightarrow \mathscr{M}^{\prime \prime} \rightarrow 0
$$

et si $\mathscr{M}$ est fuchsien le long de $Y, \mathscr{M}^{\prime}$ et $\mathscr{M}^{\prime \prime}$ le sont aussi, d'après la proposition 1.3.2, par contre si $\mathscr{M}^{\prime}$ et $\mathscr{M}^{\prime \prime}$ sont fuchsiens le long de $Y$ pour des métriques différentes, il n'est pas clair que $\mathscr{M}$ soit fuchsien.

Voyons ce que la définition précédente signifie dans le cas où $\mathscr{M}$ est défini par un seul opérateur $P$.

Lemme 2.1.2. Soit $\mathscr{M}=\mathscr{D}_{X} / \mathscr{D}_{X} P$. Alors $\mathscr{M}$ est fuchsien le long de $Y$ en $y_{0}$ si et seulement si il existe au voisinage de $y_{0}$ un système de coordonnées locales $\left(y_{1}, \ldots, y_{p}, t_{1}, \ldots, t_{q}\right)$ sur $X$ où $Y=\left\{t_{1}=\ldots=t_{q}=0\right\}$ et un entier $k$ tels que $P$ s'écrive

$$
\text { (*) } \quad P\left(y, t, D_{y}, D_{t}\right)=\sum_{\substack{|\beta|-|\alpha|=k \\: \beta \mid \leq m}} p_{\alpha \beta}(y) t^{\alpha} D_{t}^{\beta}+Q\left(y, t, D_{y}, D_{t}\right)
$$

avec
a) $Q \in V_{k-1} F_{m}\left(\mathscr{D}_{X}\right)$
b) $\forall \tau \in C^{q}-\{0\}, \sum_{\substack{|\beta|=m \\ \mid \alpha_{1}=m-k}} p_{\alpha \beta}\left(y_{0}\right) \tau^{\alpha} \bar{\tau}^{\beta} \neq 0$.

Démonstration.

Soit $S$ la sphère unité d'une métrique hermitienne de $p^{-1}\left(y_{0}\right)$ qui vérifie les conditions de la définition 2.1.1.

Choisissons des coordonnées locales $(y, t)$ de $X$ induisant les coordonnées $(y, \tau)$ sur $\Lambda$ de sorte que $S \subset \Sigma$ soit définie par $\langle\tau, \bar{\tau}\rangle=1$.

Comme d'après la Proposition 1.3.4.

$$
\begin{gathered}
C_{\Lambda \mid Y}^{1}(m)=\sigma_{\Lambda \mid Y}^{1}(P)^{-1}(0) \neq T^{*}(\Lambda \mid Y) \text { on a par définition } \\
P\left(y, t, D_{y}, D_{t}\right)=P_{0}\left(y, t, D_{t}\right)+Q\left(y, t, D_{y}, D_{t}\right) \text { où } \\
P_{0}\left(y, t, D_{t}\right)=\sum_{\substack{|\beta|-|\alpha|=k \\
|\beta| \leq m}} p_{\alpha \beta}(y) t^{\alpha} D_{t}^{\beta} \text { et }
\end{gathered}
$$

$Q \in V_{k-1} F_{m}\left(\mathscr{D}_{X}\right)$. De plus on a $\hat{\sigma}_{\Lambda}(P)=P_{0}\left(y, \tau, D_{\tau}\right)$ et la Définition 2.1.1 entraîne que $S$ est non caractéristique pour $P_{0}\left(y, \tau, D_{\tau}\right)$, c'est à dire que la condition b) est vérifiée.

q.e. d. 
Remarque. Dans le cas où $Y$ se réduit à un point de $X$ on retrouve ainsi la condition de Kashiwara-Kawai-Sjöstrand (cf. [K-K-S]).

Proposition 2.1.3. Soit $\mathscr{M}$ un $\mathscr{D}_{X}$-module cohérent fuchsien le long de $Y$ en $y_{0}$. Alors par toute section $u$ de $\mathscr{M}$ il existe un opérateur $P \in V_{0}\left(\mathscr{D}_{X}\right)$ tel que $P u=0$ et que $\mathscr{D}_{X} / \mathscr{D}_{X} P$ soit fuchsien le long de $Y$ en $y_{0}$.

\section{Démonstration.}

Fixons une métrique sur $\Sigma=p^{-1}\left(y_{0}\right)$ vérifiant la définition 2.1.1 et soit $S$ sa sphère unité.

Soit $u$ une section de $\mathscr{M}$. D'après la proposition 1.3 .2 on a

$$
C_{\Lambda \mid Y}^{1}\left(\mathscr{D}_{X} u\right) \subset C_{\Lambda \mid Y}^{1}(\mathscr{M})
$$

et donc

$$
C_{\Lambda \mid Y}^{1}\left(\mathscr{D}_{X} u\right) \cap T_{S}^{*} \Sigma \subset\{0\} .
$$

Donc, d'après la proposition 1.3.4, pour tout $\tau_{0} \in S$ il existe un voisinage ouvert $U$ de $\tau_{0}$ dans $S$ et un opérateur $P$ défini au voisinage de $y_{0}$ tels que

$$
\text { (**) } \quad C_{\Lambda \mid Y}^{1}\left(\mathscr{D}_{X} / \mathscr{D}_{X} P\right) \cap\left(T_{S}^{*} \Sigma \underset{S}{\times} U\right) \subset\{0\} .
$$

$S$ étant compacte on peut choisir un nombre fini d'ouverts $\left(U_{i}\right)_{i=1 \ldots N}$ et d'opérateurs $\left(P_{i}\right)_{i=1 \ldots N}$ définis au voisinage de $y_{0}$ tels que $\underset{i=1, \ldots, p}{\cup} U_{i}$ $=S$ et que chaque $P_{i}$ satisfasse (**) par rapport à $U_{i}$.

Considérons maintenant des coordonnées locales $(y, t)$ comme dans la démonstration du lemme précedent de sorte que $S$ soit définie par $\langle\tau, \bar{\tau}\rangle=1$.

Si $Q\left(y, t, D_{y}, D_{t}\right)=\sum_{\alpha, \beta, \gamma} a_{\alpha \beta \gamma}(y) t^{\alpha} D_{t}^{\beta} D_{y}^{\gamma}$ est un opérateur différentiel sur $X$ on notera

$$
\tilde{Q}\left(y, t, D_{y}, D_{t}\right)=\sum_{\alpha, \beta, \gamma} \overline{q_{\alpha \beta \gamma}(y)} t^{\beta} D_{t}^{\alpha} D_{y .}^{r}
$$

Comme chaque $P_{i}$ vérifie la condition $\left(^{*}\right)$ du lemme 2.1.2 avec b) remplacée par

$$
\left.\mathrm{b}^{\prime}\right) \quad \forall \tau \in U_{i}, \sum_{\substack{|\beta|=m_{i} \\|\beta|-|\alpha|=k_{i}}} p_{\alpha \beta}\left(y_{0}\right) \tau^{\alpha} \bar{\tau}^{\beta} \neq 0
$$

l'opérateur $P=\sum_{i=1, \ldots, N} \tilde{P}_{i} P_{i}$ vérifie $P u=0$ et $\mathscr{D}_{X} / \mathscr{D}_{X} P$ est fuchsien le 
long de $Y$ en $y_{0}$.

q.e. d.

Remarque. Lorsque $Y$ est une hypersurface les objets de la catégorie $R_{Y}$ définie para Kashiwara [K-3] (voir aussi Sabbah [S]) sont fuchsiens le long de $Y$.

Ainsi le lemme 4.1 .5 de $[\mathrm{K}-\mathrm{K}-2]$ montre que les modules holonômes réguliers sont fuchsiens le long de toute sous-variété de $X$.

\subsection{Théorème de comparaison pour $B_{Y \mid X}$ et $B_{Y \mid X}^{\infty}$}

Rappelons maintenant (cf. [S-K-K], [K-K-2]) que si $Y$ est une sous-variété lisse de $X$ de codimension $d$ on pose

$B_{Y \mid X}=\mathscr{H}_{[Y]}^{d}\left(\mathcal{O}_{X}\right)=\underset{m \in N}{\lim } \mathscr{E} x t_{\mathscr{O}_{X}}^{d}\left(\mathcal{O}_{X} / \mathscr{J}_{Y}^{m}, \mathcal{O}_{X}\right)$ où $\mathscr{J}_{Y}$ désigne l'idéal de définition de $Y$,

$$
\text { et } B_{Y ! X}^{\infty}=\mathscr{H}_{Y}^{d}\left(\mathcal{O}_{X}\right) \text {. }
$$

Si on se place dans un système de coordonnées locales $(y, t)$ sur $X$ où $Y=\{t=0\}$ alors $B_{Y \mid X}$ est le quotient de $\mathscr{D}_{X}$ par l'idéal engendré par $t_{1}, \ldots, t_{q}, D_{y_{1}}, \ldots D_{y_{p}}$ et $B_{Y \mid X}^{\infty}=\mathscr{D}_{X}^{\infty} \otimes_{\mathscr{D}_{X}} B_{Y \mid X}$.

Les sections de $B_{Y \mid X}^{\infty}$ sur un ouvert $U$ de $X$ s'ecrivent de manière unique

$$
u=\sum_{\alpha \in N^{q}} u_{\alpha}(y) \delta^{(\alpha)}(t)
$$

où $\delta^{(\alpha)}(t)$ désigne la classe de $D_{t}^{\alpha}$ et où les $u_{\alpha}$ sont des fonctions holomorphes sur $U \cap Y$ telles que

$$
\forall K \Subset U, \forall \varepsilon>0, \exists C_{\varepsilon}>0, \sup _{y \in K}\left|u_{\alpha}(y)\right| \leq C_{\varepsilon} \varepsilon^{|\alpha|} \frac{1}{|\alpha| !} .
$$

Alors $B_{Y \mid X}$ est le sous-ensemble de $B_{Y \mid X}^{\infty}$ des sommes finies $u=$ $\sum u_{\alpha}(y) \delta^{(\alpha)}(t)$.

Nous noterons

$$
B_{Y \mid X}(m)=\left\{u=\sum_{\alpha \in N^{n}} u_{\alpha}(y) \delta^{(\alpha)}(t), u_{\alpha}=0 \text { si }|\alpha|>m\right\} .
$$

Par suite $B_{Y \mid X}=\bigcup_{m \in N} B_{Y \mid X}(m)$.

Théorème 2.2.1. Soit $\mathscr{M}$ un $\mathscr{D}_{X}$-module cohérent fuchsien le long de $Y$ sur un ouvert $U$.

Alors le morphisme naturel 


$$
\boldsymbol{R} \mathscr{H}_{\mathrm{om}_{\mathscr{D}_{X}}}\left(\mathscr{M}, B_{Y \mid X}\right) \rightarrow \boldsymbol{R} \mathscr{H} \mathrm{om}_{\mathscr{D}_{X}}\left(\mathscr{M}, B_{Y \mid X}^{\infty}\right)
$$

est un isomorphisme sur $U$.

\section{Démonstration.}

Par une méthode désormais classique nous allons nous ramener au cas où $\mathscr{M}=\mathscr{D}_{X} / \mathscr{D}_{X} P$ pour un opérateur $P \in \mathscr{D}_{X}$. Soit donc $u_{1} \ldots u_{N}$ un système local de générateurs de $\mathscr{M}$ en $y_{0} \in U$. Suivant la définition 2.1.1 il existe une métrique hermitienne sur $\sum=p^{-1}\left(y_{0}\right)$ telle que l'on ait

$C_{\Lambda \mid Y}^{1}(\mathscr{M}) \cap T_{S}^{*} \sum \subset\{0\}$ et donc $\forall i, 1 \leq i \leq N, C_{\Lambda \mid Y}^{1}\left(\mathscr{D}_{X} u_{i}\right) \cap T_{S}^{*} \sum \subset\{0\}$ 。

D'après la proposition 2.1 .3 il existe $P_{i}$ annulant $u_{i}, P_{i} \in V_{0}\left(\mathscr{D}_{X}\right)$ tel que l'on ait encore

$$
C_{\Lambda \mid Y}^{1}\left(\mathscr{D}_{X} / \mathscr{D}_{X} P_{i}\right) \cap T_{S}^{*} \sum \subset\{0\} .
$$

Par suite $C_{\Lambda \mid Y}^{1}\left(\bigoplus_{i=1}^{N} \mathscr{D}_{X}{ }^{\prime} \mathscr{D}_{X} P_{i}\right) \cap T_{S}^{*} \sum \subset\{0\}$ et donc $\mathscr{L}=\bigoplus_{i=1}^{N} \mathscr{D}_{X} / \mathscr{D}_{X} P_{i}$ est encore un $\mathscr{D}_{X}$-module fuchsien le long de $Y$.

On obtient ainsi une suite exacte

$$
0 \rightarrow \mathscr{N} \rightarrow \mathscr{L} \rightarrow \mathscr{M} \rightarrow 0
$$

où $\mathscr{N}$ est fuchsien le long de $Y$.

On notera $\mathscr{E} x t_{\mathscr{D}_{X}}^{i}\left(\mathscr{M}, B_{Y \mid X}^{\infty}\right)=M^{j \infty}, \mathscr{E} x t_{\mathscr{D}_{X}}^{i}\left(\mathscr{M}, B_{Y \mid X}\right)=M^{j}$ et de même pour les modules $\mathscr{N}$ et $\mathscr{L}$.

On a alors le diagramme commutatif suivant dont les lignes sont exactes:

$$
\begin{aligned}
& \ldots \rightarrow M^{k-1 \infty} \rightarrow L^{k-1 \infty} \rightarrow N^{k-1 \infty} \rightarrow M^{k \infty} \rightarrow L^{k \infty} \rightarrow N^{k \infty} \rightarrow \ldots \\
& \phi_{k-1} \uparrow \quad \gamma_{k-1} \uparrow \quad \phi_{k-1} \uparrow \quad \phi_{k} \uparrow \quad \gamma_{k} \uparrow \quad \phi_{k} \uparrow \\
& \ldots \rightarrow M^{k-1} \rightarrow L^{k-1} \rightarrow N^{k-1} \rightarrow M^{k} \rightarrow L^{k} \rightarrow N^{k} \rightarrow \ldots
\end{aligned}
$$

Si le théorème est démontré dans le cas d'un opérateur, pour tout $i$ les flèches $\gamma_{i}$ sont des isomorphismes. Montrons le théorème par récurrence sur $k$.

On suppose que pour tout $l<k$ et pour tout module $\mathscr{M}$ vérifiant l'hypothèse du Théorème les morphismes $M^{l} \rightarrow M^{l \infty}$ sont des isomorphismes. Par suite dans le diagramme précédent $\phi_{k-1}$ est un isomorphisme et donc $\phi_{k}$ est injectif.

Comme $\mathscr{N}$ vérifie les hypothèses du Théorème, $\phi_{k}$ est aussi injectif 
donc $\Psi_{k}$ est surjectif, donc un isomorphisme.

Pour montrer le Théorème on peut donc supposer que $\mathscr{M}=\mathscr{D}_{X} /$ $\mathscr{D}_{X} P$, avec $P \in V_{0}\left(\mathscr{D}_{X}\right)$.

Le complexe $\boldsymbol{R} \mathscr{H}_{\mathrm{om}_{\mathscr{D}_{X}}}\left(\mathscr{M}, B_{Y \mid X}^{\infty}\right)$ est alors quasi-isomorphe à $B_{Y \mid X}^{\infty}$ $\stackrel{P}{\longrightarrow} B_{Y \mid X}^{\infty}$ tandis que le complexe $R \mathscr{H}_{\mathrm{om}_{\mathscr{D}_{X}}}\left(\mathscr{M}, B_{Y \mid X}\right)$ est quasi-isomorphe à $B_{Y \mid X} \stackrel{P}{\longrightarrow} B_{Y \mid X}$.

Nous devons donc démontrer que $P$ défini un isomorphisme de $\frac{B_{Y \mid X}^{\infty}}{B_{Y \mid X}}$ dans lui même, et nous sommes ramenés à démontrer la proposition suivante:

Proposition 2.2.2. Soit $P$ un opérateur différentiel sur $X$ d'ordre 0 pour la $V$-filtration et tel que $\left(\mathscr{D}_{X} / \mathscr{D}_{X} P\right)$ soit fuchsien le long de $Y$ en un point $y_{0}$; il existe un voisinage $U$ de $y_{0}$ et un entier $N_{0}$ tels que, pour tout $N \geq N_{0}, P$ induise un isomorphisme de $B_{Y \mid X}^{\infty} / B_{Y \mid X}(N)$ dans lui-même sur l'ouvert $U$.

Démonstration: D'aprés l'hypothèse, il existe un système de coordonnées locales $\left(y_{1}, \ldots, y_{p}, t_{1}, \ldots, t_{q}\right)$ dans lequel $y_{0}=0$,

$Y=\{(y, t) \in X \mid t=0\}$ et $P$ s'écrit :

$$
P\left(y, t, D_{y}, D_{t}\right)=P_{0}\left(y, t, D_{t}\right)+Q\left(y, t, D_{y}, D_{t}\right)
$$

avec

$$
\begin{array}{ll}
\text { 1) } & Q \in V_{-1} F_{m}\left(\mathscr{D}_{X}\right) \\
\text { 2) } & P_{0}\left(y, t, D_{t}\right)=\sum_{|\alpha|=|\beta| \leq m} p_{\alpha \beta}(y) t^{\alpha} D_{t}^{\beta}
\end{array}
$$

et

$$
\forall \tau \in C^{q}-\{0\} \sum_{|\alpha|=|\beta|=m} p_{\alpha \beta}(0) \tau^{\alpha} \bar{\tau}^{\beta} \neq 0 .
$$

L'opérateur $Q\left(y, t, D_{y}, D_{t}\right)$ peut être écrit sous la forme

$$
Q\left(y, t, D_{y}, D_{t}\right)=\sum_{\substack{|\beta|+|\gamma| \leq m \\|\alpha|>|\beta|}} q_{\alpha \beta \gamma}(y) t^{\alpha} D_{t}^{\beta} D_{y}^{\gamma}
$$

Cette somme est infinie en l'indice $\alpha$ et l'analyticité de $Q$ au voisinage de $y_{0}$ se traduit par:

$$
\begin{gathered}
\exists C>0 \forall(\alpha, \beta, \gamma, \delta) \in N^{q} \times N^{q} \times N^{p} \times N^{p},|\beta|+|\gamma| \leq m,|\alpha|>|\beta| \\
\left|\left(\frac{\partial}{\partial_{y}}\right)^{\delta} q_{\alpha \beta \gamma}(0)\right| \leq C^{|\alpha|+|\delta|+1} \delta ! .
\end{gathered}
$$


Nous poserons pour $k \in N, k \geq 1$ :

$$
P_{k}\left(y, t, D_{y}, D_{t}\right)=\sum_{\substack{|\beta|+|>| \leq m \\|\alpha|-|\beta|=k}} q_{\alpha \beta \gamma}(y) t^{\alpha} D_{t}^{\beta} D_{y}^{\gamma}
$$

on a ainsi $P=\sum_{k \geq 0} P_{k}$.

On peut encore décomposer $P_{0}$ sous la forme

$$
P_{0}\left(y, t, D_{t}\right)=P_{0}^{\prime}\left(t, D_{t}\right)+P_{0}^{\prime \prime}\left(y, t, D_{t}\right)
$$

avec $P_{0}^{\prime}\left(t, D_{t}\right)=P_{0}\left(0, t, D_{t}\right)$ et $P_{0}^{\prime \prime}\left(0, t, D_{t}\right)=0$.

On écrira

$$
P_{0}^{\prime \prime}\left(y, t, D_{t}\right)=\sum_{|\alpha|=|\beta| \leq m} q_{\alpha, \beta, 0}(y) t^{\alpha} D_{t}^{\beta}
$$

avec $q_{\alpha, \beta, 0}(0)=0$ et, puisque $q_{\alpha, \beta, 0}$ est analytique :

$$
\exists C>0 \forall \delta \in N^{p},\left|\left(\frac{\partial}{\partial_{y}}\right)^{\delta} q_{\alpha, \beta, 0}(0)\right| \leq C^{|\delta|+1} \delta ! .
$$

Notons $S=C\left[t_{1}, \ldots, t_{q}\right]$ l'espace des polynômes en $q$ variables et pour $\nu \in N, S_{\nu}$ le sous-espace de $S$ des polynômes homogènes de degré $\nu$.

Si $v=\sum v_{\alpha} t^{\alpha} \in S$, on pose $|v|=\sup _{\alpha}\left|v_{\alpha}\right|$. Rappelons un résultat de Kashiwara-Kawai-Sjöstrand [K-K-S] :

Lemme 2.2.3: Soient $\boldsymbol{S}^{2 q-1}$ la sphère unité de $\boldsymbol{C}^{q}$ et $\mu$ la mesure standard sur $\boldsymbol{S}^{2 q-1}$ normalisée par $\mu\left(\boldsymbol{S}^{2 q-1}\right)=1$.

Soit || || la norme induite sur $S$ par la norme de $L^{2}\left(S^{21-1}, \mu\right)$ :

$$
\|v\|^{2}=\int_{S^{2 q-1}} v(t) \overline{v(t)} d \mu(t) .
$$

Soit $P=\sum_{|\alpha|=|\beta| \leq m} p_{\alpha \beta} t^{\alpha} D_{t}^{\beta}$ un opérateur différentiel qui vérifie:

$$
\forall \tau \in C^{q}-\{0\} \sum_{|\alpha|=|\beta|=m} p_{\alpha \beta} \tau^{\alpha} \bar{\tau}^{\beta} \neq 0 .
$$

Il existe un entier $N_{0}$ et une constante $C>0$ tels que pour tout $k \geq N_{0}$ et tout $u \in S_{k}$ on ait:

(i) $k^{m}\|u\| \leq C\|P u\|$

(ii) $\forall \alpha \in N^{q}\left\|\tau^{\alpha} u\right\| \leq\|u\|$

(iii) $\forall \alpha \in N^{q},|\alpha| \leq k,\left\|D_{t}^{\alpha} u\right\| \leq C^{|\alpha|} \frac{k !}{(k-|\alpha|) !}\|u\|$

(iv) $C^{-k}|u| \leq\|u\| \leq C^{k}|u|$.

La partie (i) de ce lemme est montrée par [K-K-S], d'autre part 
la famille $\left(\sigma_{\alpha} t^{\alpha}\right)_{|\alpha|=k}$ pour $\sigma_{\alpha}=c_{q} \sqrt{\frac{(|\alpha|+q-1) !}{\alpha !}}$ (avec $c_{q}$ constante ne dépendant que de $q)$ est une base othonormée de $\left(S_{k},\|\|\right)$ ce qui montre facilement les points (ii), (iii) et (iv).

Suite de la démonstration de la proposition 2.2.2.

Un élement $u$ de $B_{Y \mid X}^{\infty}$ s'écrit de manière unique $u=\sum_{\alpha \in N^{q}} u_{\alpha}(y) \delta^{(\alpha)}(t)$ avec $u_{\alpha}$ holomorphe au voisinage de $y_{0}$.

A une série formelle $u=\sum_{\alpha \in N^{q}} u_{\alpha}(y) \delta^{(\alpha)}(t)$ nous associerons les polynômes $\Phi_{N}^{\delta}$ définis pour $N \in N$ et $\delta \in N^{q}$ par

$$
\Phi_{N}^{\delta}(u)=\sum_{|\alpha|=N}\left(\frac{\partial}{\partial_{y}}\right)^{\delta} u_{\alpha}(0) \tau^{\alpha}
$$

Si on pose, pour $v=\sum_{|\alpha|=N} v_{\alpha} \tau^{\alpha},|v|=\sup _{|\alpha|=N}\left|v_{\alpha}\right|$ on voit que $u=\sum u_{\alpha}$ $(y) \delta^{(\alpha)}(t)$ est un élement de $B_{Y \mid X}^{\infty}$ dans un voisinage de $y_{0}=0$ si et seulement si

$$
\exists C>0 \quad \forall \varepsilon>0 \exists C_{\varepsilon}>0 \forall N \in N \forall \delta \in N^{q}\left|\Phi_{N}^{\delta}(u)\right| \leq C_{\varepsilon} \varepsilon^{N} C^{|\delta|} \frac{\delta !}{N !} .
$$

La propriété (iv) du lemme 2.2.3 montre que dans la formule ci-dessus on peut remplacer la norme | | par la norme $\||| d u$ lemme.

D'autre part, par définition de l'action de $\mathscr{D}_{X}$ sur $B_{Y_{\mid X}}^{\infty}$ on a $t^{\alpha} \delta^{(\gamma)}(t)=(-1)^{|\alpha|} \frac{\gamma !}{(\gamma-\alpha) !} \delta^{(\gamma-\alpha)}(t)$ et $D_{t}^{\beta} \delta^{(\gamma)}(t)=\delta^{(\gamma+\beta)}(t)$ donc

a) $\Phi_{N}^{\delta}\left(t^{\alpha} u\right)=\left(-\frac{\partial}{\partial \tau}\right)^{\alpha} \Phi_{N+\mid \alpha !}^{\delta}(u)$

b) $\Phi_{N}^{\delta}\left(D_{t}^{\beta} u\right)=\tau^{\beta} \Phi_{N-|\beta|}^{\delta}(u)$

c) $\Phi_{N}^{\delta}\left(P_{0}^{\prime}\left(t, D_{t}\right) u\right)=P_{0}^{\prime}\left(-D_{\tau}, \tau\right) \Phi_{N}^{\delta}(u)$.

La condition (2.0) montre que $P_{0}^{\prime}\left(t, D_{t}\right)$ vérifie les hypothèses du lemme 2.2.3 et on observe que dans ce cas $P_{0}^{\prime}\left(-D_{\tau}, \tau\right)$ vérifie lui aussi ces hypothèses comme opérateur sur $C\left[\tau_{1}, \ldots, \tau_{q}\right]$.

On voit donc que pour tout $N \geq N_{0}\left(N_{0}\right.$ donné par le lemme) et tout $u \in B_{Y \mid X}^{\infty}$ on aura

$$
N^{m}\left\|\Phi_{N}^{\delta}(u)\right\| \leq C\left\|\Phi_{N}^{\delta}\left(P_{0}^{\prime} u\right)\right\| .
$$

L'opérateur $P_{0}^{\prime}\left(t, D_{t}\right)$ est donc inversible sur $B_{Y \mid X}^{\infty} / B_{Y \mid X}\left(N_{0}\right)$. Si $u=\sum u_{\alpha} \delta^{(\alpha)}(t)$, on posera 
$\bar{u}=\sum_{|\alpha| \geq N_{0}} u_{\alpha}(y) \delta^{(\alpha)}(t)$ et $P_{0}^{\prime-1}(u)=P_{0}^{\prime-1}(\bar{u})$ et on a une majoration $\forall N \geq N_{0}$ $\left\|\Phi_{N}^{\delta}\left(P_{0}^{-1}(u)\right)\right\| \leq C N^{-m}\left\|\Phi_{N}^{\delta}(u)\right\|$.

Soit $u \in B_{Y \mid X, y_{0}}^{\infty}$ on définit une suite $u_{n}$ de $B_{Y \mid X, y_{0}}^{\infty}$ par

$$
u_{0}=u, u_{n+1}=-\left(P_{0}^{\prime \prime}+Q\right) P_{0}^{-1}\left(u_{n}\right) \text {. }
$$

Pour montrer la proposition, nous allons montrer que la série $\sum_{n \geq 0} u_{n}$ est convergente dans $B_{Y \mid X, y_{0}}^{\infty}$ ce qui montrera que $P$ est inversible sur $B_{Y \mid X}^{\infty} / B_{Y \mid X}(N)$ pour $N \geq N_{0}$.

En fait nous allons montrer par récurrence sur $n$ que si $N_{0}$ est donné par le lemme 2.2.3 on a:

$$
\begin{aligned}
& \exists C>0, \exists \theta_{0}>0, \forall \theta, 0<\theta<\theta_{0}, \exists \varepsilon_{0}>0, \quad \forall \varepsilon, 0<\varepsilon<\varepsilon_{0}, \\
& \quad \forall n \in N, \forall N \in N, \\
& N \geq N_{0}, \forall \mu \in N^{q},\left\|\Phi_{N}^{\mu}\left(u_{n}\right)\right\| \\
& \quad \leq C_{\varepsilon} \varepsilon^{N} \theta^{n-2|\mu|} \frac{1}{N !} C^{|\mu|} \sum_{\substack{a_{1} \leq m, \ldots, a_{n} \leq m \\
a \in N^{n}}} \phi_{a}(N)(|a|+\mu) !
\end{aligned}
$$

avec $\phi_{a}(N)=\prod_{i=1}^{n} \frac{1}{(N+i)^{a_{i}}}$

(1) Si (2.4) est vérifié pourtout $n$, la série $\sum_{n \geq 0} u_{n}$ est convergente.

Fixons tout d'abord $l=|a|$ et écrivons $l=m s+r$ avec $r<m$. Comme $|a|=\sum a_{i} \leq n m$ on a $s \leq n$.

Pour $|a|=l$, la plus grande valeur de $\phi_{a}(N)$ est atteinte lorsque $a_{1}=m, \ldots, a_{s}=m, a_{s+1}=r, a_{s+2}=\ldots=a_{n}=0 \mathrm{donc}$

$$
\phi_{a}(N) \leq \phi_{a}(0) \leq \frac{1}{(s !)^{m}} \leq \frac{1}{(m s) !} 2^{[m(m+1) / 2] s} \leq C_{1}^{n} \frac{1}{l !}
$$

où $C_{1}$ ne dépend que de $m$.

$$
\sum_{a_{1} \leq m, \ldots \ldots a_{n} \leq m} \phi_{a}(N)(|a|+|\mu|) ! \leq C_{1}^{n} m^{n} \sum_{0 \leq l \leq m n} \frac{(l+|\mu|) !}{l !} \leq C_{2}^{n} 2^{|\mu|}(|\mu|) ! .
$$

On aura donc:

$$
\left\|\Phi_{N}^{\mu}\left(u_{n}\right)\right\| \leq C_{\varepsilon} \varepsilon^{N} \frac{1}{N !}\left(\frac{2 C}{\theta^{2}}\right)^{|\mu|}(|\mu|) !\left(C_{2} \theta\right)^{n} .
$$

$C_{2}$ ne dépend que de $m$ donc pour $\theta \leq \frac{1}{2 C_{2}}$, cette majoration montre que les séries $\sum_{n \geq 0} \Phi_{N}^{\mu}\left(u_{n}\right)$ sont convergentes quand $N \geq N_{0}$ et 
donc que la série $\sum_{n \geq 0} u_{n}$ est convergente.

(2) Montrons les relations (2.4) par récurrence sur $n$.

Si $u$ vérifie (2.3), il est clair qu'on a (2.4) pour $n=0$. Supposons donc (2.4) vérifiée au rang $n$ et posons $w_{n}=P_{0}^{-1}\left(u_{n}\right)$

On a $u_{n+1}=-\left(P_{0}+Q\right) u_{n}$ et $\left\|\Phi_{N}^{\mu}\left(w_{n}\right)\right\| \leq C N^{-m}\left\|\Phi_{N}^{\mu}\left(u_{n}\right)\right\|$.

$$
\Phi_{N}^{\mu}\left(u_{n+1}\right)=\sum_{\substack{\alpha, \beta, \gamma \\
\delta \leq \mu}}\left(\begin{array}{l}
\mu \\
\delta
\end{array}\right)\left(\frac{\partial}{\partial_{y}}\right)^{\mu-\delta} q_{\alpha \beta \gamma}(0) D_{\tau}^{\alpha} \tau^{\beta} \Phi_{N+|\alpha|-|\beta|}^{r+\delta}\left(w_{n}\right) \text {. }
$$

Dans cette somme on a $|\beta|+|\gamma| \leq m$ et $|\alpha| \geq|\beta|$, de plus si $|\alpha|=|\beta|$ on a $\gamma=0$ (car $P_{0}^{\prime \prime}$ est indépendant de $D_{y}$ ) et $\mu \neq \delta$ (car $\left.q_{\alpha \beta 0}(0)=0\right)$.

(La notation $\delta \leq \mu$ signifie $\forall i=1, \ldots q, \delta_{i} \leq \mu_{i}$ et $\left(\begin{array}{l}\mu \\ \delta\end{array}\right)=\frac{\mu !}{\delta !(\mu-\delta) !}$ avec $\mu !=\mu_{1} ! \ldots \mu_{q} !$.)

D'après le lemme 2.2.3 on a les majorations suivantes :

$$
\begin{aligned}
\left\|D_{\tau}^{\alpha} \tau^{\beta} \Phi_{N+|\alpha|-i \beta \mid}^{++\delta}\left(w_{n}\right)\right\| & \leq C^{|\alpha|} \frac{(N+|\alpha|-|\beta|) !}{(N-|\beta|) !}\left\|\Phi_{N+|\alpha|-|\beta|}^{\gamma+\delta}\left(w_{n}\right)\right\| \\
& \leq C^{\mid \alpha+1} \frac{(N+|\alpha|-|\beta|) !}{(N+|\alpha|-|\beta|)^{m}(N-|\beta|) !}\left\|\Phi_{N+|\alpha|-|\beta|}^{r+\delta}\left(u_{n}\right)\right\| .
\end{aligned}
$$

En utilisant l'hypothèse de récurrence et les inégalités (2.1) et (2.2) on obtient:

$$
\begin{aligned}
\left\|\Phi_{N}^{\mu}\left(u_{n+1}\right)\right\| & \leq \sum_{\substack{\alpha, \beta, \gamma \\
\delta \leq \mu}} C_{\varepsilon} \varepsilon^{N+|\alpha|-|\beta|} \theta^{n}\left(\frac{C}{\theta^{2}}\right)^{|\gamma|+|\delta|} \sum_{a_{1} \leq m, \ldots, a_{n} \leq m} \phi_{a}(N+|\alpha|-|\beta|) \\
\cdot(|\alpha|+|\gamma|+|\delta|) ! C^{|\alpha|+1} C^{|\alpha|+; \mu|-| \delta \mid+1} & \\
\cdot & \frac{1}{(N+|\alpha|-|\beta|)^{m}(N-|\beta|) !}\left(\begin{array}{c}
\mu \\
\delta
\end{array}\right)(\mu-\delta) !
\end{aligned}
$$

La somme ci-dessus se décompose en $A_{1}+A_{2}$ où $A_{1}$ est la somme des termes tels que $|\alpha|=|\beta|$ (donc $\gamma=0, \delta<\mu$ ) et $A_{2}$ la somme pour $|\alpha|>|\beta|$.

$$
\begin{aligned}
A_{1}= & \sum_{\substack{|\alpha|=|\beta| \leq m \\
\delta<\mu}} C_{\varepsilon} \varepsilon^{N} \frac{\theta}{N !}^{n-2|\mu|}\left(\theta^{2}\right)^{|\mu|-|\delta|} C^{2|\alpha|+|\mu|+2} \\
& \cdot \sum_{a_{1} \leq m, \ldots, a_{n} \leq m} \phi_{a}(N)(|a|+|\delta|) ! \frac{\mu !}{\delta !}
\end{aligned}
$$

Comme 


$$
\sum_{\delta<\mu} \theta^{2(|\mu|-|\delta|)} \frac{(|a|+|\delta|) !}{\delta !} \leq \frac{(|a|+|\mu|) !}{\mu !} \frac{\theta}{\left(1+\theta^{2}\right)^{p}} .
$$

on obtient:

$$
\begin{gathered}
A_{1} \leq C_{\varepsilon} \varepsilon^{N} \frac{\theta^{n-2|\mu|+1}}{N !} C^{|\mu|} \sum_{\substack{a_{1} \leq m, \ldots, a_{n} \leq m \\
a_{n+1}=0}} \phi_{a}(N)(|a|+|\mu|) ! \cdot \\
\cdot \sum_{|\alpha|=|\beta| \leq m} C^{2|\alpha|+2} \frac{\theta}{\left(1+\theta^{2}\right)^{p}}
\end{gathered}
$$

Il existe $\theta_{0}$ ne dépendant que de la dimension $p$ de $Y$, de $m$ et de $C$ tel que si $\theta \leq \theta_{0}$,

$$
\frac{\theta}{\left(1+\theta^{2}\right)^{p}} \sum_{|\alpha|=|\beta| \leq m} C^{2|\alpha|+2} \leq \frac{1}{2} .
$$

Considérons maintenant $A_{2}$, on a donc $|\alpha|>|\beta|$ et $|\beta|+|\gamma| \leq m$

$$
\frac{N !}{(N-|\beta|) !} \frac{1}{(N+|\alpha|-|\beta|)^{m}} \leq \frac{N^{|\beta|}}{(N+1)^{m}} \leq \frac{1}{(N+1)^{m-|\beta|}}
$$

On pose $\tilde{a}_{1}=m-|\beta|, \tilde{a}_{2}=a_{2}, \ldots, \tilde{a}_{n+1}=a_{n}$ donc $|\tilde{a}|=|a|+m-|\beta| \geq$ $|a|+|\gamma|$ et comme $|\alpha|-|\beta| \geq 1$ on $a$ :

$$
\begin{aligned}
& \frac{N !}{(N-|\beta|) !} \frac{1}{(N+|\alpha|-|\beta|)^{m}} \phi_{a}(N+|\alpha|-|\beta|) \leq \frac{1}{(N+1)^{m-|\beta|}} \\
& \phi_{a}(N+|\alpha|-|\beta|) \leq \phi_{\tilde{a}}(N) \\
& A_{2} \leq \sum_{\alpha, \beta, \gamma} C_{\varepsilon} \varepsilon^{N+|\alpha|-|\beta|} \frac{\theta^{n-2|\mu|}}{N !} C^{|\mu|} \sum_{\tilde{a}_{1} \leq m, \ldots, \tilde{a}_{n+1} \leq m} \phi_{\tilde{a}}(N) \cdot \\
& \cdot C^{2|\alpha|+2} \sum_{\delta \leq \mu} \theta^{2 \uparrow|\mu|-|\delta|)} \frac{\mu !}{\delta !}(|\tilde{a}|-m+|\beta|+|\gamma|+|\delta|) !
\end{aligned}
$$

(Dans la suite nous écrirons $a$ pour $\tilde{a}$ ). On a :

$$
\begin{gathered}
\frac{(|a|-m+|\beta|+|\gamma|+|\delta|) !}{\delta !} \leq \frac{(|a|+|\delta|) !}{\delta !} \leq \frac{(|a|+|\mu|) !}{\mu !} \\
\sum_{\delta \leq \mu} \theta^{2(|\mu|)-|\delta|)} \leq 2 \text { si } \theta \leq \theta_{0} \leq 1 .
\end{gathered}
$$

On obtient donc :

$$
A_{2} \leq C_{\varepsilon} \varepsilon^{N} \frac{\theta^{n-2|\mu|+1}}{N !} C^{|\mu|} \sum_{a_{1} \leq m, \ldots, a_{n+1} \leq m} \phi_{a}(N)(|a|+|\mu|) ! S(\varepsilon)
$$

$\operatorname{avec} S(\varepsilon)=\frac{2}{\theta} \sum_{\substack{\alpha, \beta|>| \beta|,| \beta|+| \alpha|\leq m\\| \alpha|\alpha|+2}} C^{2|\alpha|-|\beta|}$.

Pour tout $C>0$ et tout $\theta>0$, il existe $\varepsilon_{1}(\theta)>0$ tel que 


$$
\forall \varepsilon<\varepsilon_{1}(\theta), S(\varepsilon)<\frac{1}{2} .
$$

Donc pour $\theta \leq \theta_{0}$ et $\varepsilon \leq \inf \left(\varepsilon_{1}(\theta), \varepsilon_{0}\right)$ on aura :

$$
\left\|\Phi_{N}^{\mu}\left(u_{n+1}\right)\right\| \leq A_{1}+A_{2} \leq C_{\varepsilon} \varepsilon^{N} \frac{\theta^{n+1-2|\mu|}}{N !} C_{a_{1} \leq m, \ldots, a_{n+1} \leq m}^{|\mu|} \phi_{a}(N)(|a|+|\mu|) !
$$

ce qui termine la démonstration de la proposition 2.2.2.

\section{3. Théorèmes de comparaison pour $\mathcal{O}_{X}$ et $\mathcal{O}_{X \hat{\mid} Y}$}

Nous allons à présent démontrer un théorème de comparaison concernant le faisceau $\mathcal{O}_{X}$ des fonctions holomorphes et le complété formel de $\mathcal{O}_{X}$ le long d'une sous-variété.

Si $Y$ est une sous-variété lisse de $X$, nous noterons comme précédemment $\mathscr{J}_{Y}$ l'idéal de $\mathcal{O}_{X}$ des fonctions holomorphes nulles sur $Y$.

Le complété formel de $\mathcal{O}_{X}$ le long de $Y$ est le faisceau :

$$
\mathcal{O}_{X \hat{Y} Y}=\stackrel{\lim }{\longleftarrow} \mathcal{O}_{X} / \mathscr{J}_{Y}^{k} \text {. }
$$

Théorème 2.3.1. Soit $\mathscr{M}$ un $\mathscr{D}_{X}$ module cohérent fuchsien le long de $Y$ en $y_{0} \in Y$. Il existe un voisinage $U$ de $y_{0}$ dans $Y$ sur lequel on $a$ :

$$
\left.\boldsymbol{R} \mathscr{H} \operatorname{om}_{\mathscr{D}_{X}}\left(\mathscr{M}, \mathcal{O}_{X}\right)\right|_{U}=\left.\boldsymbol{R} \mathscr{H} \operatorname{om}_{\mathscr{D}_{X}}\left(\mathscr{M}, \mathcal{O}_{X \hat{Y} Y}\right)\right|_{v} .
$$

Nous démontrerons ce théorème directement en nous ramenant, suivant la même méthode que pour ce théorème 2.2.1, au cas d'un seul opérateur, c'est à dire à la proposition suivante:

Proposition 2.3.2. Soit $P$ un opérateur différentiel sur $X$, d'ordre 0 pour la $V$-filtration, tel que $\mathscr{D}_{X} / \mathscr{D}_{X} P$ soit fuchsien le long de $Y$ en un point $y_{0}$. Il existe un voisinage $U$ de $y_{0}$ et un entier $k_{0} \geq 0$ tel que pour tout $k \geq k_{0}, P$ induise sur $U$ des isomorphismes $\mathscr{J}_{Y}^{k} \rightarrow \mathscr{J}_{Y}^{k}$ et $\mathscr{J}_{Y}^{k} \mathcal{O}_{X \hat{\uparrow}} \rightarrow$ $\mathscr{J}_{Y}^{k} \mathcal{O}_{X \hat{Y} Y}$.

Remarque : Cette proposition a été démontrée par Kashiwara-KawaiSjöstrand dans le cas ou $Y$ est un point et par Oshima [O] lorsque $Y$ est une hypersurface. Nous suivrons ici la démonstration de [K-K-S] (en utilisant en particulier le lemme 2.2.3). 
Démonstration: Nous nous plaçons dans un système de coordonnées locales $\left(y_{1}, \ldots, y_{p}, t_{1}, \ldots, t_{q}\right)$ où $P$ se décompose comme dans la démonstration de la proposition 2.2.2 et s'écrit donc

$$
P=\sum_{k \geq 0} P_{k}, P_{k} \text { opérant de } \mathscr{J}_{Y}^{l} \text { dans } \mathscr{J}_{Y}^{l+k} \text {. }
$$

On note encore $S=C\left[t_{1}, \ldots, t_{q}\right]$ et $S_{N}$ le sous-espace de $S$ des polynômes homogènes de degré $N$.

On désigne par $\mathcal{O}_{Y, 0}$ le germe en $y_{0}=0$ du faisceau $\mathcal{O}_{Y}$ des fonctions holomorphes sur $Y, \mathscr{S}=\mathcal{O}_{Y, 0} \bigotimes_{C} S=\mathcal{O}_{Y, 0}\left[t_{1}, \ldots t_{q}\right]$ et $\mathscr{S}_{N}=\mathcal{O}_{Y, 0} \bigotimes_{c} S_{N}$.

Si $u(y, t)$ est un élément de $\mathscr{S}_{N}$ on peut l'écrire en développant ses coefficients en série de Taylor en 0 :

$$
u(y, t)=\sum_{\alpha \in N} u_{\alpha}(t) y^{\alpha} \text {. }
$$

Pour tout $\rho>0$ on pose:

$$
\|u\|_{\rho}=\sum_{\alpha \in N^{p}}\left\|u_{\alpha}\right\| \rho^{|\alpha|}
$$

où $\left\|u_{\alpha}\right\|$ est la norme sur $S_{N}$ définie dans le lemme 2.2.3.

Nous noterons $\mathscr{S}_{N}(\rho)$ le sous-espace de $\mathscr{S}_{N}$ des éléments $u$ tels que $\|u\|_{\rho}<+\infty$-il est clair que $\mathscr{S}_{N}=\cup_{\rho>0} \mathscr{S}_{N}(\rho)$ (cette définition s'applique également à $\left.\mathcal{O}_{Y, 0}=\mathscr{S}_{0}\right)$.

Les éléments de $\mathcal{O}_{X \hat{Y} Y}\left(\right.$ resp. de $\mathscr{J}_{Y}^{k} \mathcal{O}_{X \hat{Y} Y}$ ) sont les séries formelles $u=\sum_{\nu \geq 0} u_{\nu}$ (resp. $u=\sum_{\nu>k} u_{\nu}$ ) avec $u_{\nu} \in \mathscr{S}_{\nu}$ pour tout $\nu_{\text {. }}$

Il est clair (vu le (iv) du lemme 2.2.3) qu'un élément $u=\sum u_{\nu}$ $\operatorname{de} \mathcal{O}_{X \hat{Y} Y}\left(\right.$ resp. de $\mathscr{J}_{Y}^{k} \mathcal{O}_{X \hat{Y}}$ ) est dans $\mathcal{O}_{X, y_{0}}\left(\right.$ resp. $\left.\mathscr{J}_{Y, y_{0}}^{k}\right)$ si et seulement si :

$$
\exists \rho>0, \exists C>0, \forall \nu \geq 0,\left\|u_{\nu}\right\|_{\rho} \leq C^{\nu+1}
$$

Lemme 2. 3. 3: L'opérateur $P=\sum_{\mu \geq 0} P_{\mu}$ vérifie,

1) $\exists C_{1}>0, \exists \rho_{0}>0, \exists k_{0}, \forall \rho>0, \rho \leq \rho_{0}, \forall k \in N, k \geq k_{0}, \forall u \in \mathscr{S}_{k}(\rho)$,

$$
k^{m}\|u\|_{\rho} \leq C_{1}\left\|P_{0} u\right\|
$$

2) $\exists \rho_{0}>0, \forall \rho>0, \rho \leq \rho_{0}, \exists C_{2}>0, \exists C_{3}>0, \forall \mu>0, \forall k \geq 0$,

$$
\begin{aligned}
& \forall u \in \mathscr{S}_{k}(\rho), \forall(s, t), \frac{1}{2}<s<t<1, \\
& \left\|P_{\mu} u\right\|_{s \rho} \leq C_{2}^{m} C_{3}^{\mu+1} \sum_{p=0}^{m} \frac{k ! p !}{(k-m+p) !} \frac{1}{(t-s)^{p}}\|u\|_{t \rho} .
\end{aligned}
$$


Démonstration: D'après le lemme 2.2.3 on a, pour tout $\rho>0$, tout $u \in \mathscr{S}_{k}(\rho)$ et tout $(\alpha, \beta) \in \boldsymbol{N}^{q} \times \boldsymbol{N}^{q}$ :

$$
\left\|t^{\alpha} D_{t}^{\beta} u\right\|_{\rho} \leq C^{|\beta|} \frac{k !}{(k-|\beta|) !}\|u\|_{\rho} .
$$

D'autre part la formule de Cauchy montre (cf. [B-K]) que:

$$
\forall(s, t) 1 / 2<s<t,\left\|D_{y}^{\alpha} u\right\|_{s \rho} \leq C^{|\alpha|}|\alpha| !\left(\frac{1}{t-s}\right)^{|\alpha|}\|u\|_{t_{\rho}}
$$

$$
\text { L'opérateur } P_{\mu} \text { s'écrit } P_{\mu}=\sum_{\substack{|\beta|+|\gamma| \leq m \\|\alpha|-|\beta|=\mu}} q_{\alpha \beta}(y) t^{\alpha} D_{t}^{\beta} D_{y}^{\gamma}
$$

et d'après (2.1) il existe $\rho_{0}>0$ et $C_{0}>0$ tel que pour $\rho \leq \rho_{0}$ on ait

$$
\left\|q_{\alpha \beta \gamma}\right\| \leq C_{0}^{|\alpha|+1} \text {. }
$$

On obtient donc immédiatement le point 2) du lemme. D'autre part $P_{0}$ se décompose comme précédemment sous la forme:

$$
P_{0}\left(y, t, D_{t}\right)=P_{0}^{\prime}\left(t, D_{t}\right)+P_{0}^{\prime \prime}\left(y, t, D_{t}\right)
$$

avec $P_{0}^{\prime}\left(t, D_{t}\right)=P_{0}\left(0, t, D_{t}\right)$.

L'opérateur $P_{0}^{\prime}$ vérifie les hypothèses du lemme 2.2.3 donc:

$$
\begin{aligned}
& \exists k_{0} \in \boldsymbol{N}, \exists C_{0}>0 \exists \rho_{0}>0 \quad \forall \rho \leq \rho_{\nu} \forall k \in N, k \geq k_{0}, \\
& \forall u \in \mathscr{S}_{k}(\rho), k^{m}\|u\|_{\rho} \leq C_{0}\left\|P_{0}^{\prime} u\right\|_{\rho} .
\end{aligned}
$$

De plus $P_{0}^{\prime \prime}\left(y, t, D_{t}\right)=\sum_{|\alpha|=|\beta| \leq m} \tilde{p}_{\alpha \beta}(y) t^{\alpha} D_{t}^{\beta}$ avec $\tilde{p}_{\alpha \beta}(0)=0$ donc $\forall \varepsilon>0 \exists \delta>0 \forall \rho<\delta,\left\|\tilde{p}_{\alpha \beta}\right\|_{\rho} \leq \varepsilon$ et donc si $u \in \mathscr{S}_{k}(\rho)$ on aura :

$$
\left\|P_{0}^{\prime \prime} u\right\|_{\rho} \leq \varepsilon \sum_{|\alpha|=\mid \beta ! \leq m} C^{|\beta|} \frac{k !}{(k-|\beta|) !}\|u\|_{\rho} \leq C^{\prime} \varepsilon \frac{k !}{(k-m) !}\|u\|_{\rho} \leq C^{\prime} \varepsilon k^{m}\|u\|_{\rho} .
$$

Si on choisit $\varepsilon=\frac{1}{2 C^{\prime} C_{0}}$ on obtient :

$$
2 C_{0}\left\|P_{0} u\right\|_{\rho} \geq 2 C_{0}\left\|P_{0}^{\prime} u\right\|_{\rho}-\frac{1}{\varepsilon C^{\prime}}\left\|P^{\prime \prime} u\right\|_{\rho} \geq k^{m}\|u\|_{\rho}
$$

q. e. d.

Fin de la démonstration de la proposition 2.3.2.

Le lemme 2.3.3 montre qu'il existe $k_{0}$ tel que pour $k \geq k_{0}, P_{0}$ soit bijectif de $\mathscr{S}_{k}$ dans lui même.

On en déduit immédiatement que pour $k \geq k_{0} P$ est injectif sur $\mathscr{J}_{Y}^{k} \mathcal{O}_{X \hat{Y} Y}$ et donc aussi sur $\mathscr{J}_{Y}^{k}$.

Montrons que $P$ est surjectif. 
Si $u=\sum_{\nu \geq k} u_{\nu}$ est un élément de $\mathscr{J}_{Y}^{k} \mathcal{O}_{X \hat{Y} Y}$ et si $v=P u$ on a $v=\sum_{\nu \geq k} v_{\nu}$ avec $v_{\nu}=\sum_{\mu \geq 0} P_{\mu} u_{\nu-\mu}$.

L'équation $P \quad u=v$ est donc équivalente a :

$$
\forall j \geq k, \quad P_{0} u_{j}=v_{j}-\sum_{\nu<j} P_{j-\nu} u_{j}
$$

Pour $k \geq k_{0}, P_{0}$ est bijectif sur $\mathscr{S}_{k}$ donc on peut toujours résoudre les équations ci dessus et $P$ est surjectif de $\mathscr{J}_{Y}^{k} \mathcal{O}_{X \mid Y} \operatorname{sur} \mathscr{J}_{Y}^{k} \mathcal{O}_{X \mid Y}$. Pour montrer que $P$ est surjectif $\mathscr{J}_{Y}^{k} \rightarrow \mathscr{J}_{Y}^{k}$ il suffit de montrer que si $v \in \mathscr{J}_{Y}^{k}$ alors $u \in \mathscr{J}_{Y}^{k}$.

Soit $v=\sum_{\nu \geq k} v_{\nu} \in \mathscr{J}_{Y}^{k}$, il existe $\rho>0$ et $D>0$ tels que

$$
\forall \nu \geq k,\left\|v_{\nu}\right\|_{\rho} \leq\left(\frac{D}{2^{m}}\right)^{\nu+1}
$$

et donc pour $1 / 2<s<1,\left\|v_{\nu}\right\|_{s \rho} \leq\left(\frac{D}{2^{m}}\right)^{\nu+1} \leq D^{\nu+1}\left(\frac{1}{1-s}\right)^{m \nu}$. Soit $u=\sum u_{j}$ tel que $P u=v$.

Montrons par récurrence sur $j$ que pour tout $j$ on a:

$$
\forall s, \frac{1}{2}<s<1,\left\|u_{j}\right\|_{s \rho} \leq C^{j+1}\left(\frac{1}{1-s}\right)^{m s}
$$

où $C$ est une constante à déterminer.

Pour $j<k, u_{j}=0$ donc la relation est vérifiée. Supposons cette rélation vérifiée pour tout $\nu<j$.

Si $P u=v$ on a $P_{0} u_{j}=v_{j}-\sum_{\nu<j} P_{j-\nu} u_{\nu}$

D'après le lemme 2.3.3 on a:

$$
\forall s, \frac{1}{2}<s<1,\left\|u_{\|}\right\|_{s \rho} \leq C_{1} j^{-m}|| P_{0} u \|_{s \rho}
$$

et

$$
\left\|P_{J-\nu} u_{\nu}\right\|_{s \rho} \leq C_{2}^{m} C_{3}^{j-\nu+1} \sum_{p=0}^{m} \frac{\nu ! p !}{(\nu-m+p) !} \frac{1}{(t-s)^{p}}\left\|u_{\nu}\right\|_{t \rho}
$$

et donc d'après l'hypothèse de récurrence :

$$
\begin{aligned}
& \left\|u_{j}\right\|_{s \rho} \leq C_{0} j^{-m}\left[\left\|v_{j}\right\|_{s \rho}+\sum_{\nu<j}\left\|P_{j-\nu} u_{\nu}\right\|_{s \rho}\right] \\
& \left\|P_{j-\nu} u_{\nu}\right\|_{s \rho} \leq C_{2}^{m} C_{3}^{j-\nu+1} \sum_{p=0}^{m} \frac{\nu ! p !}{(\nu-m+p) !} \frac{1}{(t-s)^{p}} C^{\nu+1}\left(\frac{1}{1-t}\right)^{m \nu} .
\end{aligned}
$$

Cette inegalité est vraie pour tout $t$ tel que $\frac{1}{2}<s<t<1$, or 


$$
\inf _{s<t<1}\left(\frac{1}{t-s}\right)^{p}\left(\frac{1}{1-t}\right)^{m \nu}=\left(\frac{1}{1-s}\right)^{p+m \nu} \frac{(p+m \nu)^{p+m \nu}}{p^{p}(m \nu)^{m \nu}}
$$

donc

$$
\begin{gathered}
\left\|P_{j-\nu} u_{\nu}\right\|_{s \rho} \leq C_{2}^{m} C_{3}^{j-\nu+1} C^{\nu+1}\left(\frac{1}{1-s}\right)^{m(\nu+1)} \sum_{p=0}^{m} \frac{\nu ! p !}{(\nu-m+p) !} \frac{(p+m \nu)^{p+m \nu}}{p^{p}(m \nu)^{m \nu}} \\
\text { Or } \frac{\lambda !}{(\lambda-m+p) !} \leq \lambda^{m-p} \\
\frac{(m \lambda+p)^{m \lambda+p}}{(m \lambda)^{m \lambda} p^{p}}=\left(1+\frac{p}{m \lambda}\right)^{m \lambda}\left(1+\frac{m \lambda}{p}\right)^{p} \leq\left(\frac{2 m^{2}}{p}\right)^{p} e^{p}
\end{gathered}
$$

donc $\sum_{p=0}^{m} \frac{\nu ! p !}{(\nu-m+p) !} \frac{(p+m \nu)^{p+m}}{p^{p}(m \nu)^{m \nu}} \leq \nu^{m} a$ où $a$ ne dépend que de $m$.

$$
\begin{aligned}
\sum_{\nu<j}\left\|P_{j-\nu} u_{\nu}\right\|_{s \rho} & \leq C_{2}^{m} a j^{m}\left(\frac{1}{1-s}\right)^{m j} \sum_{\nu<j} C_{3}^{j-\nu+1} C^{\nu+1} \\
& \leq C_{2}^{m} a j^{m} C_{3}^{2} \frac{C^{j+1}}{C-C_{3}}\left(\frac{1}{1-s}\right)^{m j}
\end{aligned}
$$

et donc

$$
\|u,\|_{s} \leq\left(\frac{1}{1-s}\right)^{m j}\left[C_{0} D^{\prime+1}+a C_{0} C_{2}^{m} \frac{C_{3}^{2}}{C-C_{3}} C^{\jmath}\right]
$$

et la relation de récurrence est vérifiée dès que

$$
C>2 C_{0} D \text { et } C>C_{3}+2 a C_{0} C_{2}^{m} C_{3}^{2} \text {. }
$$

q. e. d.

Soit $\mathscr{M}$ un $\mathscr{D}_{X}$-module cohérent. On note $\mathscr{M}^{*}=\boldsymbol{R} \mathscr{H}$ om $\mathscr{D}_{X}\left(\mathscr{M}, \mathscr{D}_{X}\right)-$ c'est un complexe de $\mathscr{D}_{X}$-modules à droite. Les constructions menant à la définition 2.1.1 étant encore valables dans la catégorie des $\mathscr{D}_{X}$-modules à droite cohérents, on définit de la même manière la notion de $\mathscr{D}_{X}$-module à droite fuchsien le long de $Y$ et on a :

Proposition 2. 3.4. Soit $\mathscr{M}$ un $\mathscr{D}_{X}$-module cohérent. Alors:

i) $\quad C_{A \mid Y}^{1}\left(\mathscr{E}^{2} x t_{\mathscr{D}_{X}}^{\prime}\left(\mathscr{M}, \mathscr{D}_{X}\right)\right) \subset C_{\Lambda \mid Y}^{1}(\mathscr{M})$.

ii) Si $\mathscr{M}$ est fuchsien le long de $Y$ alors $\mathscr{M}^{*}$ est un complexe dont les groupes de cohomologie sont fuchsiens le long de $Y$.

\section{Démonstration.}

ii) Résulte immédiatement de i). 
i) 1) Considérons d'abord le cas où $\mathscr{M}=\mathscr{D}_{X} / \mathscr{D}_{X} P$. Alors $\mathscr{M}^{*} \simeq$ $\mathscr{D}_{X} / P \mathscr{D}_{X}$ et le résultat est évident.

2) Dans le cas général, soit donné localement un système de générateurs $u_{1} \ldots u_{N}$ de $\mathscr{M}$.

Soit $x^{*} \in T^{*}(\Lambda \mid Y)$ tel que $x^{*} \notin C_{A \mid Y}^{1}(\mathscr{M})$.

Il s'agit donc de montrer que pour tout $j$

$$
x^{*} \notin C_{\Lambda \mid Y}^{1}\left(\mathscr{E} x t_{\mathscr{D}_{X}}^{j}\left(\mathscr{M}, \mathscr{D}_{X}\right)\right) .
$$

On a une suite exacte

$$
0 \leftarrow \mathscr{M} \leftarrow \bigoplus_{j=1}^{N} \mathscr{D}_{X} / \mathscr{D}_{X} P_{j} \leftarrow \mathscr{N} \leftarrow 0
$$

où $P_{j} u_{j}=0, \mathscr{L}=\bigoplus_{j=1}^{N} \mathscr{D}_{X} / \mathscr{D}_{X} P_{j}$ et $x^{*} \notin C_{\Lambda_{\mid Y}}^{1}(\mathscr{L})$.

Pour tout $j \neq 1, \mathscr{E} x t \stackrel{j}{\mathscr{D}}_{X}\left(\mathscr{L}, \mathscr{D}_{X}\right)=0$ et d'après 1)

$$
x^{*} \notin C_{\Lambda \mid Y}^{1}\left(\mathscr{E} x t_{\mathscr{D}_{X}}^{1}\left(\mathscr{L}, \mathscr{D}_{X}\right)\right) \text {. }
$$

D'autre part on a les suites exactes

$$
\begin{aligned}
& 0 \rightarrow \mathscr{E} x t_{\mathscr{D}_{X}}^{1}\left(\mathscr{M}, \mathscr{D}_{X}\right) \rightarrow \mathscr{E} x t_{\mathscr{D}_{X}}^{1}\left(\mathscr{L}, \mathscr{D}_{X}\right) \rightarrow \\
& \mathscr{E} x t_{\mathscr{D}_{X}}^{1}\left(\mathscr{N}, \mathscr{D}_{X}\right) \rightarrow \mathscr{E}^{x} t_{\mathscr{D}_{X}}^{2}\left(\mathscr{M}, \mathscr{D}_{X}\right) \rightarrow 0
\end{aligned}
$$

et

$$
\forall j \geq 3 \quad 0 \rightarrow \mathscr{E} x t_{\mathscr{D}_{X}}^{j-1}\left(\mathscr{N}, \mathscr{D}_{X}\right) \rightarrow \mathscr{E} x t_{\mathscr{D}_{X}}^{j}\left(\mathscr{M}, \mathscr{D}_{X}\right) \rightarrow 0
$$

On déduit de $\left(^{*}\right)$ que $x^{*} \notin C_{\Lambda \mid Y}^{1}\left(\mathscr{E}^{x} t_{\mathscr{D}_{X}}^{1}\left(\mathscr{M}, \mathscr{D}_{X}\right)\right)$ et donc $x^{*} \notin C_{\Lambda \mid Y}^{1}$ $\left(\mathscr{E} x t_{\mathscr{D}_{X}}^{1}\left(\mathcal{N}, \mathscr{D}_{X}\right)\right)$. On raisonne alors par récurrence sur $j$.

q. e. d.

Remarques:

1-Les théorèmes de "comparaison" 2.2.1 et 2.3.1 restent valables pour les $\mathscr{D}_{X}$-modules $\mathscr{M}$ qui sont extension de modules fuchsiens le long de $Y$, c'est à dire, pour les modules pour qui il existe une suite exacte $0 \rightarrow \mathscr{N}^{\prime} \rightarrow \mathscr{M} \rightarrow \mathscr{N}^{\prime \prime} \rightarrow 0$ où $\mathscr{N}^{\prime}$ et $\mathscr{N}^{\prime \prime}$ sont fuchsiens le long de $Y$. 2 -Les théorèmes 2.2.1 et 2.3.1 restent valables si on remplace $\mathscr{M}$ par un complexe $\dot{\mathscr{M}}$ de $\mathscr{D}_{X}$-modules cohérents dont les groupes de cohomologie $\mathscr{H}^{j}(\dot{\mathscr{M}})$ sont nuls pour $|j|$ assez grand et sont fuchsiens le long de $Y$. En particulier si $\mathscr{M}$ est fuchsien ils sont vrais pour le

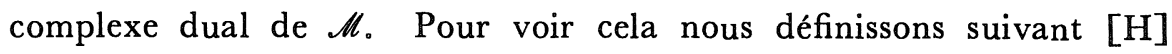
les foncteurs "troncature" $\sigma_{\geq n}$ et $\sigma_{\geq n}^{\prime}$ où $n \in N$ par 


$$
\begin{aligned}
& \sigma_{\geq n}^{\prime}(\mathscr{M})=0 \longrightarrow \text { coker } d^{n-1} \stackrel{d^{n}}{\longrightarrow} \mathscr{M}^{n+1} \stackrel{d^{n+1}}{\longrightarrow} \mathscr{M}^{n+2} \longrightarrow \ldots \\
& \sigma_{\geq n}(\dot{\mathscr{M}})=0 \longrightarrow \operatorname{Im} d^{n} \stackrel{i}{\longrightarrow} \mathscr{M}^{n+1} \stackrel{d^{n+1}}{\longrightarrow} \mathscr{M} \stackrel{n+2}{\longrightarrow} \ldots
\end{aligned}
$$

Alors on a $H^{i}\left(\sigma_{\geq n}(\dot{\mathscr{M}})\right)=\left\{\begin{aligned} & H^{i}(\dot{\mathscr{M}}) \text { pour } i \geq n+1 \\ & 0 \text { pour } i<n+1\end{aligned}\right.$

et $H^{i}\left(\sigma_{\geq n}^{\prime}(\dot{\mathscr{M}})\right)=\left\{\begin{aligned} H^{i}(\dot{\mathscr{M}}) & \text { pour } i \geq n \\ 0 & \text { pour } i<n\end{aligned}\right.$. De plus on a un triangle

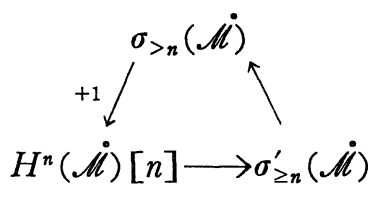

et on peut donc raisonner par récurrence décroissante sur $n$.

\section{§ 3. Applications}

Reprenons les notations du paragraphe précédent.

Soit $X$ une variété analytique complexe et $Y$ une sous-variété lisse de $X$. Soit $\mathscr{J}_{Y}$ l'idéal de définition de $Y$. Rappelons que l'on note

$$
\mathscr{D}_{Y \rightarrow X}=\mathscr{D}_{X} / \mathscr{J}_{Y} \mathscr{D}_{X} ; \text { c'est un }\left(\mathscr{D}_{Y}, \mathscr{D}_{X}\right) \text {-bimodule }
$$

On a $\mathscr{D}_{Y \rightarrow X}=B_{Y \mid Y \times X} \underset{\mathscr{O}_{X}}{\otimes} \Omega_{X}$ où $\Omega_{X}$ désigne le faisceau des formes différentielles holomorphes de degré maximum et où l'on identifie $Y$ au graphe de l'injection $Y \longrightarrow X$. Soit $\mathscr{D}_{X}^{\infty}$ le faisceau des opérateurs différentiels d'ordre fini ou infini sur $X$. On note $\mathscr{D}_{Y \rightarrow X}^{\infty}=\mathscr{D}_{Y \rightarrow Y}{\underset{\mathscr{D}}{X}}_{X} \mathscr{D}_{X}^{\infty}$ $=\mathscr{D}_{X}^{\infty} / \mathscr{J}_{Y} \mathscr{D}_{X}^{\infty}$.

On a de même $\mathscr{D}_{Y \rightarrow X}^{\infty}=B_{Y \mid Y \times X}^{\infty} \otimes \mathscr{O}_{X} \Omega_{X}$ et on pose encore $\mathscr{D}_{X \leftarrow Y}=$ $B_{Y \mid Y \times X} \underset{\mathcal{O}_{Y}}{\otimes} \Omega_{Y}$ et $\mathscr{D}_{X \leftarrow Y}^{\infty}=B_{Y \mid Y \times X}^{\infty} \underset{\mathscr{O}_{Y}}{\bigotimes} \Omega_{Y}$. Si $\mathscr{M}$ est un $\mathscr{D}_{X}$-module cohérent, le système induit par $\mathscr{M}$ sur $Y$ est par définition le complexe $\dot{\mathscr{M}}_{Y}=$ $\mathscr{D}_{Y \rightarrow X} \stackrel{\sim}{\otimes}_{\mathscr{D}_{X}}^{u} \mathscr{M}=\mathcal{O}_{Y} \stackrel{\mathcal{O}}{X}_{X}^{u} \mathscr{M}$. Si $\mathscr{L}$ est un $\mathscr{D}_{X}^{\infty}$-module on pose de même

$$
\dot{\mathscr{L}}_{Y}=\mathscr{D}_{Y \rightarrow X}^{\infty} \bigotimes_{\mathcal{O}_{X}^{\infty}}^{u} \mathscr{L} \simeq \mathcal{O}_{Y} \bigotimes_{\mathcal{O}_{X}}^{u} \mathscr{L}
$$

Remarquons que si $\mathscr{M}$ est un $\mathscr{D}_{X}$-module cohérent fuchsien le long de $Y$ alors $\mathscr{M}$ est elliptique le long de $Y$ au sens de [L-Sch] (cf. aussi [Sch]) et donc $\mathscr{M}_{Y}$ est à cohomologie cohérente sur $\mathscr{D}_{Y}$. 


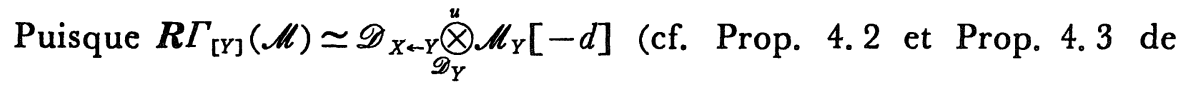
[K-2]) on en déduit que $\boldsymbol{R} \Gamma_{[Y]}(\mathscr{M})$ est à cohomologie cohérente sur $\mathscr{D}_{X}$.

\section{1. Comparaison des cohomologies d'un système fuchsien}

Si $\mathscr{M}$ est $\mathscr{D}_{X}$-module cohérent nous noterons $\mathscr{M}^{\infty}=\mathscr{D}_{X}^{\infty} \mathscr{\mathscr { D }}_{X} \mathscr{M}$.

Théorème 3.1.1. Soit $\mathscr{M}$ un $\mathscr{D}_{X}$-module fuchsien sur un ouvert $U$ de Y. Alors le morphisme naturel

$$
\left(\boldsymbol{R} \Gamma_{[Y]}(\mathscr{M})\right)^{\infty} \rightarrow \boldsymbol{R} \Gamma_{Y}\left(\mathscr{M}^{\infty}\right) \text { est un isomorphisme. }
$$

Remarque. Dans le cas où $\mathscr{M}$ est holonôme singulier régulier ce théorème a été démontré par Kashiwara-Kawai [K-K-2] (voir aussi Mebkhout [M]).

\section{Démonstration.}

Par une méthode analogue à celle du théorème 2.2.1 on peut se ramener au cas où $\mathscr{M}=\mathscr{D}_{X} / \mathscr{D}_{X} P$ avec $P$ fuchsien le long de $Y$ et $P \in V_{0}\left(\mathscr{D}_{X}\right)$. Il faut donc démontrer que les complexes

$$
0 \longrightarrow \mathscr{D}_{X}^{\infty}{\underset{\mathscr{D}}{X}}_{X} R \Gamma_{[Y]}(\mathscr{D})_{X} \stackrel{P}{\longrightarrow} \mathscr{D}_{X}^{\infty}{\underset{\mathscr{D}}{X}}_{X} R \Gamma_{[Y]}\left(\mathscr{D}_{X}\right) \longrightarrow 0
$$

et

$$
0 \longrightarrow R \Gamma_{Y}\left(\mathscr{D}_{X}^{\infty}\right) \stackrel{P}{\longrightarrow} R \Gamma_{Y}\left(\mathscr{D}_{X}^{\infty}\right) \longrightarrow 0
$$

sont quasi-isomorphes.

Soit $d$ la codimension de $Y$ dans $X$.

Considerons la suite d'immersions fermées de variétés $Y \subset Y \times X \subset$ $X \times X$ et notons $Z=Y \times X, \mathscr{X}=X \times X$. Rappelons que $\mathscr{D}_{X}=B_{X \mid X \times X} \bigotimes_{\mathcal{O}_{X}}^{\bigotimes}$ $\Omega_{X}$ (cf. $\left.[\mathrm{S}-\mathrm{K}-\mathrm{K}]\right)$. Comme le théorème est de nature locale on peut donc identifier $\mathscr{D}_{X}$ et $B_{X \mid X \times X}$.

On a alors $\boldsymbol{R} \Gamma_{[Y]}\left(\mathscr{D}_{X}\right) \simeq \boldsymbol{R} \Gamma_{[Y]}\left(\boldsymbol{R} \Gamma_{[X]}\left(\mathcal{O}_{X \times X}\right)[n]\right) \simeq$ $R \Gamma_{[Y]}\left(\boldsymbol{R} \Gamma_{[Z]}\left(\mathcal{O}_{X \times X}\right)\right)[n] \simeq \boldsymbol{R}_{[Y]}\left(B_{Z \mid \mathscr{X}}\right)[n-d]$,

et de même 


$$
\boldsymbol{R} \Gamma_{Y}\left(\mathscr{D}_{X}^{\infty}\right) \simeq R \Gamma_{Y}\left(B_{Z \mid \mathscr{X}}^{\infty}\right)[n-d] .
$$

Par la première projection de $X \times X$ dans $X$, l'opérateur $P$ s'identifie à un opérateur de $\mathscr{D}_{X \times X}$ fuchsien le long de $Z$. D'après la proposition 2.2.2 il existe donc un entier $N_{0} \geq 0$ tel que pour tout $N \geq N_{0}$ le morphisme de complexes

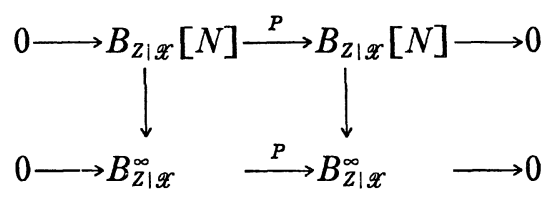

soit un quasi-isomorphisme. On a donc un quasi-isomorphisme

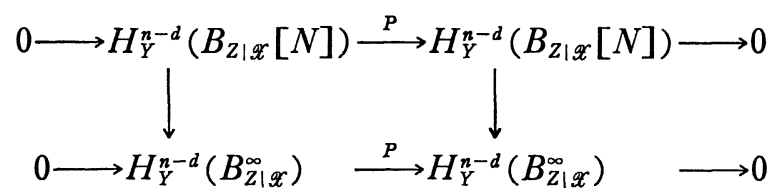

Identifions $B_{Z \mid \mathscr{X}}[N]$ à $\mathcal{O}_{z} \bigotimes_{C} S_{N}$ où $S_{N}$ désigne comme au $\S 2$ le $\boldsymbol{C}$ espace vectoriel des polynômes à $d$-variables de degré inférieur ou égal à $N$ et $B_{Z \mid \mathscr{X}}$ à $\mathcal{O}_{z} \underset{\mathrm{C}}{\otimes} S$ où $\mathrm{S}=\underset{N}{\stackrel{\lim }{\longrightarrow}} S_{N}$ est le $\boldsymbol{C}$-espace vectoriel des polynômes à $d$-variables.

On a alors

$$
\begin{aligned}
& \boldsymbol{R} \Gamma_{Y}\left(B_{Z \mid \mathscr{X}}[N]\right) \simeq R \Gamma_{Y}\left(\mathcal{O}_{Z}\right) \underset{C}{\otimes} S_{N} \text { et d'autre part }
\end{aligned}
$$

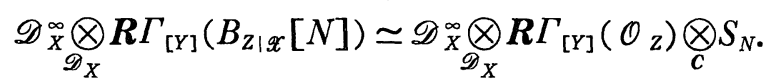

Vérifions donc que $\mathscr{D}_{X}^{\infty} \underset{\mathscr{D}_{X}}{\otimes} \boldsymbol{R} \Gamma_{[Y]}\left(\mathcal{O}_{Z}\right) \simeq \boldsymbol{R} \Gamma_{Y}\left(\mathcal{O}_{Z}\right)$. Puisque $X$ et $Z$ sont transversales dans $X \times X$ et que $X \cap Z=Y$ on a

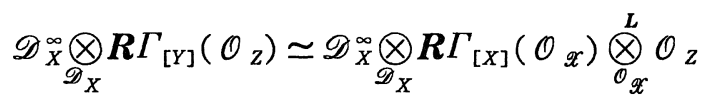

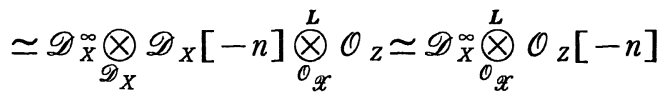

$$
\begin{aligned}
& \simeq \boldsymbol{R} \Gamma_{X}\left(\mathcal{O}_{\mathscr{X}}\right){\underset{\mathcal{O}}{\mathscr{X}}}_{\mathbb{L}}^{\mathrm{O}} \mathcal{O}_{Z} \simeq \boldsymbol{R} \Gamma_{Y}\left(\mathcal{O}_{Z}\right) \text {. }
\end{aligned}
$$

Il reste à remarquer que $\underset{N}{\stackrel{\lim }{\longrightarrow}} \mathscr{D}_{X}^{\infty} \mathscr{D}_{X} \underset{R}{R} \Gamma_{[Y]}\left(\mathcal{O}_{Z}\right) \underset{C}{\otimes} S_{N} \simeq \mathscr{D}_{X}^{\infty}{\underset{\mathscr{D}}{X}}_{X} \boldsymbol{R} \Gamma_{[Y]}$ $\left(B_{Z \mid \mathscr{X}}\right)$.

q. e. d. 


\section{2. Systèmes induits}

Théorème 3.2.1. Soit $\mathscr{M}$ un $\mathscr{D}_{X}$-module cohérent fuchsien le long de $Y$ sur un ouvert $U$ de $Y$. Alors le morphisme naturel:

$$
\mathscr{D}_{Y}^{\infty} \underset{\mathscr{D}_{Y}}{\otimes}\left(\mathscr{D}_{Y \rightarrow X} \stackrel{L}{\underset{\mathscr{D}}{\otimes}} \mathscr{M}\right) \longrightarrow \mathscr{D}_{Y \rightarrow X}^{\infty} \underset{\mathscr{D}_{X}}{\otimes} \mathscr{L}
$$

est un isomorphisme $\left(c^{\prime}\right.$ est à dire $\left.\left(\mathscr{M}^{\infty}\right)_{Y} \simeq\left(\mathscr{M}_{Y}\right)^{\infty}\right)$.

Démonstration.

D'après le théorème 3.1 .1 on a

$$
\begin{aligned}
& \left(\boldsymbol{R} \Gamma_{[Y]}(\mathscr{M})\right)^{\infty} \simeq \boldsymbol{R} \Gamma_{Y}\left(\mathscr{M}^{\infty}\right) \text { et donc }
\end{aligned}
$$

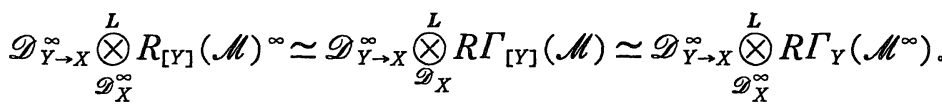

Or comme nous l'avons vu plus haut $\boldsymbol{R} \Gamma_{[Y]}(\mathscr{M}) \simeq \mathscr{D}_{X \leftarrow Y} \bigotimes_{\mathscr{D}_{Y}} \mathscr{M}_{Y}[-d]$ donc

$$
\begin{aligned}
& \mathscr{D}_{Y \rightarrow X}^{\infty} \underset{\mathscr{D}_{X}}{\otimes} R \Gamma_{[Y]}(\mathscr{M}) \simeq \mathscr{D}_{Y \rightarrow X}^{\infty}{\underset{\mathscr{D}}{X}}_{X} \mathscr{D}_{X \rightarrow Y} \bigotimes_{\mathscr{D}_{Y}} \mathscr{M}_{Y}[-d] \simeq \\
& \mathscr{D}_{Y}^{\infty}{\underset{\mathscr{D}}{Y}}_{Y} \mathscr{M}_{Y} \simeq\left(\mathscr{M}_{Y}\right)^{\infty} .
\end{aligned}
$$

( $d$ désignant la codimension de $Y$ dans $X$ ).

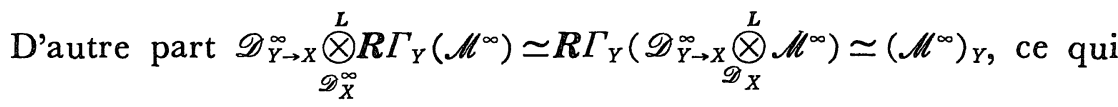
termine la démonstration. q. e. d.

Soit $\mathscr{M}$ un $\mathscr{D}_{X}$-module. Alors on a un isomorphisme canonique $\left.\boldsymbol{R} \mathscr{H} \mathrm{om}_{\mathscr{D}_{X}}\left(\mathscr{M}, \mathcal{O}_{Y \hat{Y} X}\right)\right|_{Y} \simeq \boldsymbol{R} \mathscr{H} \mathrm{om}_{\mathscr{D}_{Y}}\left(\mathscr{M}_{Y}, \mathcal{O}_{Y}\right)$ (Il suffit de vérifier ce résultat dans le cas $\mathscr{M}=\mathscr{D}_{X}$ ).

Le Théorème 2.3.1 permet donc d'obtenir le théorème de Cauchy :

Théorème 3.2.2. Soit $\mathscr{M}$ un $\mathscr{D}_{X}$-module fuchsien le long de $Y$; alors le morphisme naturel

$$
\left.\boldsymbol{R} \mathscr{H} \mathrm{om}_{\mathscr{D}_{X}}\left(\mathscr{M}, \mathcal{O}_{X}\right)\right|_{Y} \longrightarrow \boldsymbol{R} \mathscr{H} \mathrm{om}_{\mathscr{D}_{Y}}\left(\mathscr{M}_{Y}, \mathcal{O}_{Y}\right)
$$

est un isomorphisme.

Ce même théorème 2.3.1 entraîne la proposition: 
Proposition 3. 2.3. Soit $\mathscr{M}$ un $\mathscr{D}_{X}$-module fuchsien le long de $Y$. Alors le morphisme naturel

$$
\boldsymbol{R} \Gamma_{Y}\left(\Omega_{X} \stackrel{L}{\mathscr{D}_{X}} \mathscr{M}\right) \longrightarrow \Omega_{Y} \stackrel{L}{\bigotimes_{\mathscr{P}_{Y}}} \mathscr{M}_{Y}[-d]
$$

est un isomorphisme.

\section{Démonstration.}

On a

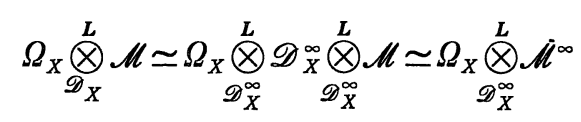

et donc

$$
\begin{aligned}
& R \Gamma_{Y}\left(\Omega_{X} \stackrel{\stackrel{L}{\otimes}}{\otimes} \mathscr{M}\right) \simeq R \Gamma_{Y}\left(\Omega_{X} \underset{\mathscr{D}_{X}^{\infty}}{\stackrel{L}{\otimes}} \mathscr{M}^{\infty}\right) \\
& \simeq \Omega_{X} \underset{\mathscr{D}_{X}^{\infty}}{\stackrel{L}{\bigotimes} R} \Gamma_{Y}\left(\mathscr{M}^{\infty}\right) \simeq \Omega_{X} \underset{\mathscr{D}_{X}^{\infty}}{\bigotimes^{L}}\left(\boldsymbol{R} \Gamma_{[Y]}(\mathscr{M})\right)^{\infty} \\
& \simeq \Omega_{X} \underset{\mathscr{D}_{X}}{\stackrel{L}{\otimes}} \boldsymbol{R} \Gamma_{[Y]}(\mathscr{M})
\end{aligned}
$$

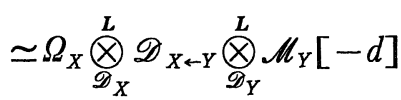

$$
\begin{aligned}
& \simeq \Omega_{Y} \stackrel{L}{\mathscr{Q}_{Y}} \mathscr{M}_{Y}[-d] \text {. }
\end{aligned}
$$

q. e. d.

Les résultats du $\S 2$ permettent encore de donner un théorème de Cauchy pour $B_{Y \mid X}^{\infty}$ :

Si $\mathscr{M}$ est un $\mathscr{D}_{X}$-module cohérent (ou un complexe de $\mathscr{D}_{X}$-modules cohérents à cohomologie cohérente) on note comme précédement:

$$
\mathscr{M}^{*} \simeq \boldsymbol{R} \mathscr{H} \operatorname{om}_{\mathscr{D}_{X}}\left(\mathscr{M}, \mathscr{D}_{X}\right) \text {. }
$$

Le complexe induit sur $Y$ par $\mathscr{M}^{*}$ est par définition $\mathscr{M}_{Y}^{*} \simeq \mathscr{M}^{*}{\stackrel{\bigotimes}{\mathscr{X}_{X}} \mathscr{L}}_{X \leftarrow Y}$. Enfin $\left(\mathscr{M}_{Y}^{*}\right)^{*}$ est le dual de $\mathscr{M}_{Y}^{*}$, c'est à dire $\boldsymbol{R} \mathscr{H}_{\mathrm{om}_{\mathscr{D}_{Y}}}\left(\mathscr{M}_{Y}^{*}, \mathscr{D}_{Y}\right)$.

Théorème 3.2.4. Soit $\mathscr{M}$ un $\mathscr{D}_{X}$-module fuchsien le long de Y. Alors on $a$

$$
\boldsymbol{R} \mathscr{H} \mathrm{om}_{\mathscr{D}_{X}}\left(\mathscr{M}, B_{Y \mid X}^{\infty}\right) \mid Y \simeq \boldsymbol{R} \mathscr{H} \mathrm{om}_{\mathscr{D}_{Y}}\left(\left(\mathscr{M}_{Y}^{*}\right)^{*}, \mathcal{O}_{Y}\right)
$$




\section{Démonstration.}

Nous utiliserons le lemme suivant:

\section{Lemme 3.2.5.}

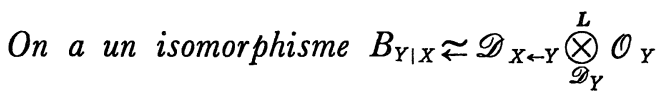

\section{Démonstration.}

Soit $p: Y \times X \rightarrow X$ la deuxième projection; d'après $[\mathrm{S}-\mathrm{K}-\mathrm{K}]$ on a un morphisme d'image directe

$$
p^{*}: B_{Y \mid Y \times X}{\underset{O}{O_{Y}}}_{\underset{X}{X}} \Omega_{Y} \simeq \mathscr{D}_{X \leftarrow Y} \longrightarrow B_{Y_{\mid X}}
$$

Puisque $\mathscr{O}_{Y}$ est un sous anneau de $\mathscr{D}_{Y}$ on obtient un morphisme

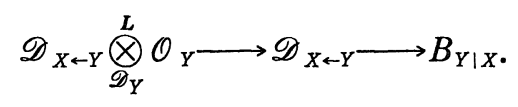

Pour montrer que ce morphisme est un isomorphisme on peut se placer dans un système de coordonnées locales $\left(y_{1}, \ldots y_{p}, t_{1} \ldots t_{q}\right)$ sur $X$ où $Y$ est définie par $t_{1}=\ldots=t_{q}=0$. Alors $\mathscr{D}_{X \leftarrow Y} \simeq \frac{\mathscr{D}_{X}}{\mathscr{D}_{X} t_{1}+\ldots+\mathscr{D}_{X} t_{q}}$ et $\mathscr{D}_{X+Y} \stackrel{\bigotimes}{\mathscr{Q}}_{Y}^{L} \mathscr{O}_{Y} \simeq \frac{\mathscr{D}_{X}}{\mathscr{D}_{X} t_{1}+\ldots+\mathscr{D}_{X} t_{q}+\mathscr{D}_{X} D_{y_{1}} \ldots+\mathscr{D}_{X} D_{y_{p}}} \simeq B_{Y \mid X}$.

q. e. d.

Démonstration du théorème 3.2.4.

D'après le théorème 2.2.1 on a

$$
\boldsymbol{R} \mathscr{H} \mathrm{om}_{\mathscr{D}_{X}}\left(\mathscr{M}, B_{Y \mid X}^{\infty}\right) \simeq \boldsymbol{R} \mathscr{H} \mathrm{om}_{\mathscr{D}_{X}}\left(\mathscr{M}, B_{Y \mid X}\right) .
$$

Par ailleurs d'après le lemme 3.2.5 on a

$$
B_{Y \mid X} \simeq \mathscr{D}_{X \leftarrow Y} \bigotimes_{\mathscr{D}_{Y}}^{L} \mathcal{O}_{Y}
$$

donc

$$
\begin{aligned}
& \boldsymbol{R} \mathscr{H} \operatorname{om}_{\mathscr{D}_{X}}\left(\mathscr{M}, B_{Y \mid X}\right) \simeq \boldsymbol{R} \mathscr{H} \operatorname{om}_{\mathscr{D}_{X}}\left(\mathscr{M}, \mathscr{D}_{X}\right) \underset{\mathscr{D}_{X}}{\stackrel{L}{\otimes}} B_{Y \mid X}
\end{aligned}
$$

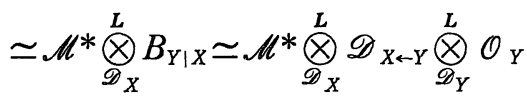

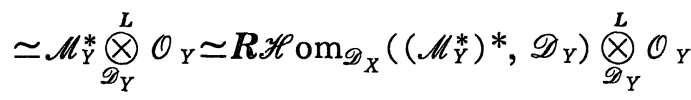




$$
\simeq \boldsymbol{R} \mathscr{H} \operatorname{om}_{\mathscr{D}_{Y}}\left(\left(\mathscr{M}_{Y}^{*}\right)^{*}, \mathcal{O}_{Y}\right)
$$

q. e. d.

\section{Bibliographie}

[B-K] Boutet de Monvel, L., Kree, P., Pseudodifferential operators and Gevrey classes, Ann. Inst. Fourier Grenoble, T-26 (1976), 81-140.

[H] Hartshorne, R., Residues and Duality, Lecture Notes in Maths., Springer, 20 (1966).

[K-1] Kashiwara, M., On the maximally overdetermined systems of linear differential equations I, Publ. RIMS, Kyoto Univ., 10 (1975), 563-579.

[K-2] On the holonomic systems of linear differential equations, II, Inventiones Math., 49 (1979), 121-135.

[K-3] Vanishing cycle sheaves and holonomic systems of differential equations, Springer Lect. Notes in Math., $\mathbf{n}^{\circ} 1016$ (1983).

[K-K-1] Kashiwara, M., Kawai, T., Second microlocalisation and asymptotic expansions, Lecture Notes in Physics, Springer, 126 (1980), 21-56.

[K-K-2] , On the holonomic systems of microdifferential equations, III, Publ. RIMS, Kyoto Univ., 17 (1981), 813-979.

[K-K-S] Kashiwara, M., Kawai, T., Sjostrand, J., On a class of linear partial differential equations whose formal solutions always converge, Arkiv für Math., 17, n ${ }^{\circ}$ (1979), 83-91.

[L] Laurent, Y., Théorie de la deuxième microlocalisation dans le domaine complexe, Progress in Math. Birkhäuser, 1985.

[L-Sch] Laurent, Y., Schapira, P., Images inverses des modules différentiels, Compositio Mathematica, 61 (1987), 229-251.

[M] Mebkhout, Z., Une équivalence de catégories et une autre équivalence de catégories, Compositio mathematica, vol 51, n ${ }^{\circ}$ (1984), pp. 55-62 et pp. 63-68.

[M.F.] Monteiro Fernandes, T., Problème de Cauchy microdifférentiel et théorèmes de propagation-in "Géometrie et analyse microlocal”, Astérisque, n 140-141 (1986), 135220.

[O] Oshima, T., A Definition of boundary values of solutions of partial differential equations with regular singularities, Publ. RIMS, Kyoto Univ., 19 (1983), 1203-1230.

[R] Ramis, J. P., Variations sur le thème GAGA, Lecture Notes in Math., Springer, 694 (1978), 228-289.

[S] Sabbah, C., $\mathscr{D}$-modules et cycles évanescents, apparu.

[Sch] Schapira, P., Microdifferential systems in the complex domain, Grundlehren der mathmatischen Wissenchaften, 269, Springer-Verlag.

[S-K-K] Sato, M., Kashiwara, M., Kawai, T., Hyperfunctions and pseudo-differential equations, Lecture Notes in Math., Springer-Verlag, 287 (1973), 265-529. 
